\title{
Vitamin D status and hypertension: a review
}

This article was published in the following Dove Press journal:

Integrated Blood Pressure Control

8 April 2015

Number of times this article has been viewed

\section{Liang $\mathrm{Ke}^{\prime}$ \\ Rebecca S Mason ${ }^{2}$ \\ Maina Kariuki ${ }^{3}$ \\ Elias Mpofu' \\ Kaye E Brock ${ }^{2}$}

'Faculty of Health Sciences, ${ }^{2}$ Faculty of Medicine, University of Sydney, ${ }^{3}$ Biostatistical Officer Training Program, NSW Ministry of Health, Sydney, NSW, Australia
Correspondence: Kaye E Brock

Faculty of Medicine, University of Sydney, Sydney 2006, NSW, Australia

Tel +6I 2 935। 6544

Fax +6I 2 935। 2058

Email kaye.brock@sydney.edu.au
Abstract: Vitamin D is a steroid prohormone synthesized in the skin following ultraviolet exposure and also achieved through supplemental or dietary intake. While there is strong evidence for its role in maintaining bone and muscle health, there has been recent debate regarding the role of vitamin D deficiency in hypertension based on conflicting epidemiological evidence. Thus, we conducted a scoping systematic literature review and meta-analysis of all observational studies published up to early 2014 in order to map trends in the evidence of this association. Mixed-effect meta-analysis was performed to pool risk estimates from ten prospective studies $(\mathrm{n}=58,262)$ (pooled risk for incident hypertension, relative risk $[R R]=0.76(0.63-0.90)$ for top vs bottom category of 25-hydroxyvitamin D [25OHD]) and from 19 cross-sectional studies $(n=90,535)$ (odds ratio $[\mathrm{OR}]=0.79(0.73-0.87))$. Findings suggest that the better the assessed quality of the respective study design, the stronger the relationship between higher $25 \mathrm{OHD}$ levels and hypertension risk $(\mathrm{RR}=0.67(0.51-0.88)$; $\mathrm{OR}=0.77(0.72-0.89))$. There was significant heterogeneity among the findings for both prospective and cross-sectional studies, but no evidence of publication bias was shown. There was no increased risk of hypertension when the participants were of older age or when they were vitamin D deficient. Younger females showed strong associations between high 25OHD levels and hypertension risk, especially in prospective studies $(\mathrm{RR}=0.36(0.18-0.72) ; \mathrm{OR}=0.62(0.44-0.87))$. Despite the accumulating evidence of a consistent link between vitamin $\mathrm{D}$ and blood pressure, these data are observational, so questions still remain in relation to the causality of this relationship. Further studies either combining existing raw data from available cohort studies or conducting further Mendelian analyses are needed to determine whether this represents a causal association. Large randomized controlled trials are also needed to determine whether vitamin supplementation may be beneficial in the prevention or the treatment of hypertension.

Keywords: 25OHD, high blood pressure, meta-analysis, prospective, cross-sectional, blood pressure

\section{Introduction}

Despite recent downward trends in cardiovascular disease (CVD) mortality rates, ${ }^{1}$ CVD still represents $50 \%$ of noncommunicable disease deaths worldwide, ${ }^{2}$ and there is an exponential increase in CVD incidence in lower- and middle-income countries. ${ }^{3}$ Hypertension, which is also increasing, ${ }^{4}$ is one of the primary modifiable risk factors for CVD, and as such, any new modifiable risk factor associated with prevention of this condition is important for public health measures. Obesity and lack of physical activity (PA) and increased salt intake are well-known and studied modifiable environmental factors associated with hypertension. In recent times, vitamin D deficiency has also been postulated to be such a factor. ${ }^{5-8}$ 
Vitamin D is a steroid prohormone synthesized in the skin following ultraviolet exposure. It is also achieved through supplemental or dietary intake. While there is strong evidence for its role in maintaining bone and muscle health, ${ }^{9}$ there has been recent debate regarding the role of vitamin $\mathrm{D}$ deficiency in CVD conditions ${ }^{10,11}$ based on conflicting epidemiological evidence.

There is a growing body of evidence from animal ${ }^{12}$ and clinical studies ${ }^{13}$ that vitamin D-mediated reduction of hypertension involves increased activation of the renin-angiotensin-aldosterone system, which is the main regulator of electrolyte and volume homeostasis that contributes to the development of arterial hypertension.

Epidemiologically, cross-sectional studies have consistently shown associations of hypertension with vitamin D deficiency as measured by the level of 25-hydroxyvitamin D (25OHD) (in nanomoles per litre) in the blood, ${ }^{7}$ and the most recent meta-analyses of prospective studies ${ }^{8}$ have also found this association to be persistent over time. However, metaanalysis results from randomized controlled trials (RCTs) (the gold standard of epidemiological studies) of 25OHD blood levels and hypertension have been null. ${ }^{14}$ This discrepancy may well be due to the small size and specialized population samples of the RCTs.

Due to the continued interest and debate in this area, we conducted a scoping systematic literature review and meta-analysis of all observational studies published up to early 2014 in order to map trends in the evidence on the association between blood vitamin $\mathrm{D}$ levels and the risk of hypertension.

\section{Methods}

For this systematic review, studies concerned with hypertension and 25OHD were identified using a predefined protocol and in accordance with Preferred Reporting Items for Systematic Reviews and Meta-Analyses (PRISMA) statement. ${ }^{15}$ Unlike previous reviews, ${ }^{7,8}$ we included studies that varied by culture of respondents for broader perspective (Supplementary material).

\section{Data source and study search}

We systematically conducted independent searches of Scopus and PubMed databases for published articles from January 1, 2007 until February 22, 2014. Identical search strategies were applied for each database search with combined terms of “25OHD”, "25-hydroxyvitamin D”, "vitamin D”, "hypertension", "vitamin D supplementation (cholecalciferol [vitamin D3], ergocalciferol [vitamin D2])", "systolic blood pressure (SBP)", or "diastolic blood pressure (DBP)". Reference lists of retrieved articles were automatically imported into Endnote X5 and manually scanned for relevant review articles. Duplicated references from two databases were detected by Endnote $X 5$. In order to proceed further in the review process, retrieved articles' abstracts were read by two independent reviewers (KB and LK). Only those abstracts that were related to $25 \mathrm{OHD}$ levels and hypertension were kept for full-text review and meta-analysis. Disagreements were resolved by a consensus or by reference with co-authors: statistician (MK) and/or vitamin D physiologist (RSM). We restricted the search to human studies and those published in English.

\section{Study selection}

As a very recent meta-analysis has been published on RCTs, ${ }^{14}$ only observational (cross-sectional and prospective) studies were included in this meta-analysis. For these observational studies, the independent risk factor was plasma or serum vitamin D levels measured as 25OHD in blood, and the outcome was hypertension or SBP and DBP. Dietary vitamin D was not included in this systematic review. Figure 1 shows a flowchart of the data extraction.

We restricted this meta-analysis (Tables 1-4 and Figures $2 \mathrm{~A}$ and $3 \mathrm{~A}$ ) to studies which recruited healthy adult study populations (aged greater than 18 years) from the general population. Prospective studies were included if they had at least 1 year of follow-up, with 25OHD levels measured at baseline, and if the results were reported categorically as a relative risk (RR) or an odds ratio (OR) with $95 \%$ confidence intervals (CIs).

Studies were excluded if the study sample was not recruited from the general population (ie, participants who had hypertension at baseline or participants with conditions that may influence vitamin D metabolism such as obese population groups ${ }^{16}$ or autoimmune diseases ${ }^{17}$ ). Exclusions applied as well if there was more than one study that investigated the same data (eg, The National Health and Nutrition Examination Survey (NHANES) ${ }^{18}$ duplicates were excluded).

Table 5 contains other extracted studies that reported associations with linear 250HD levels. These studies were included in the systematic review results and discussion but not in the formal meta-analysis mapping.

\section{Data extraction and quality assessment}

The following data were extracted from each study:

- first authors' last name, year of publication, study name, year of study conducted, and quality score;

- country of studies' origin and study population; 


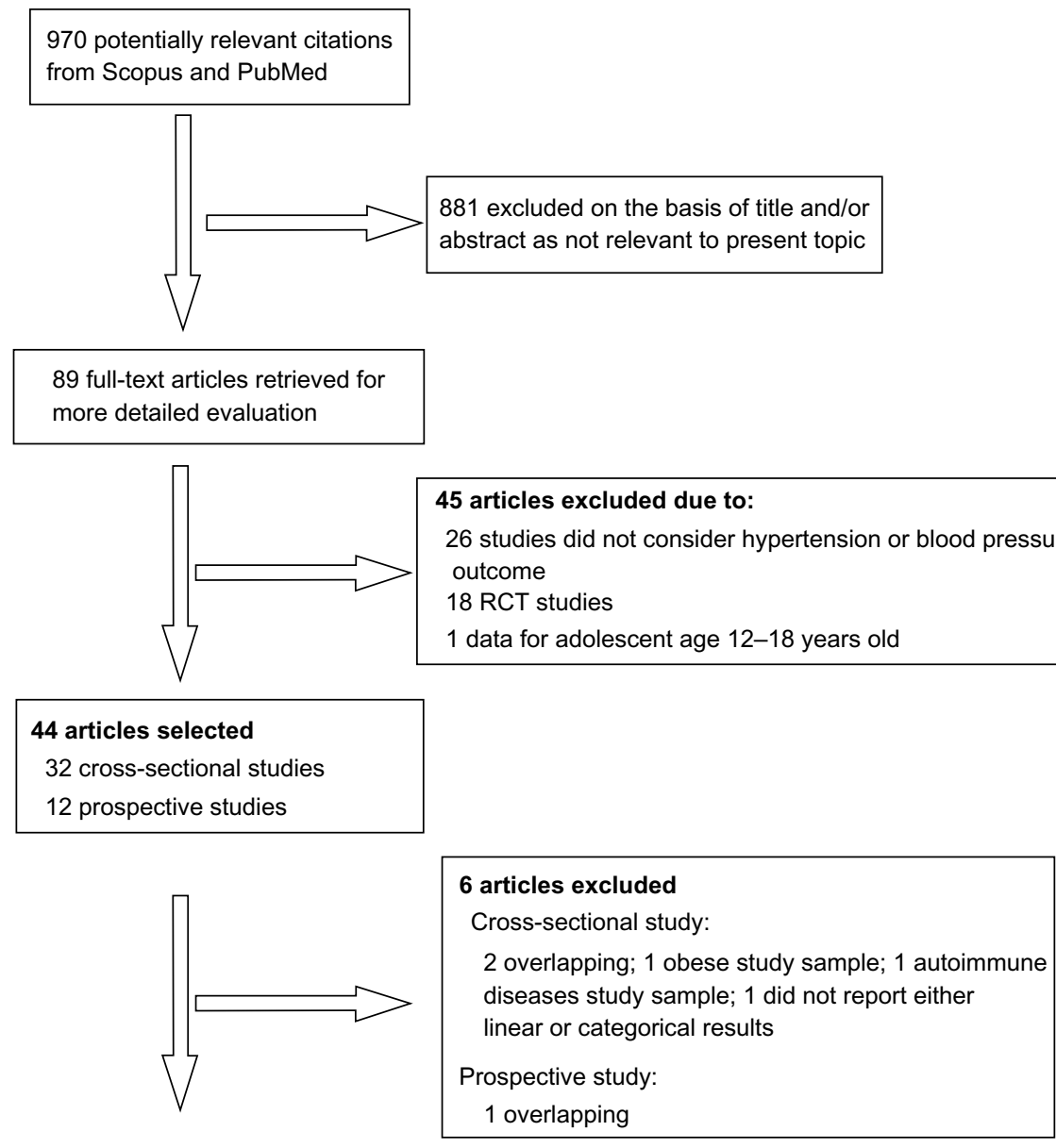

29 studies included in meta-analysis

19 cross-sectional studies ( 1 that reported linear results was included in Table 5)

10 prospective studies (4 that reported linear results were included in Table 5)

9 studies ( 8 cross-sectional studies and 1 prospective study) only reported linear

results included in Table 5

Figure I Flowchart of meta-analysis data extraction.

Abbreviation: RCT, randomized controlled trial.

- gender and age;

- sample size (number of cases of hypertension in the case of prospective studies);

- baseline 25OHD as either percent of vitamin D deficiency or mean of 25OHD and vitamin D assay method;

- 25OHD categories;

- mode of hypertension measurement;

- risk estimates (RRs or ORs) with corresponding CIs for blood 25OHD concentrations; and

- confounders measured and taken account for in analysis.

Study quality score was assessed based on the nine-star Newcastle-Ottawa Scale (NOS) ${ }^{10}$ using predefined criteria namely selection (population representativeness), comparability (adjustment for confounders such as age, gender, body mass index [BMI], PA, ethnicity, season, antihypertensive treatment, and diabetes status used), and ascertainment of outcome. In this predefined scoring method, a maximum of four points were given for selection, two points for comparability, and three points for outcome. Nine points on the NOS reflect the highest study quality. ${ }^{19}$

\section{Data synthesis and analysis}

Mixed-effect meta-analysis ${ }^{20}$ was performed to pool risk estimates for both observational and prospective studies. For both studies, we synthesized RR (for prospective studies) or OR (for cross-sectional studies), and 95\% CI. These estimate compare the lowest vitamin D status as defined by categorical levels of 25OHD (as the reference group) to the highest vitamin D levels in order to estimate the risk of association with hypertension (primary endpoint). 
Table I Characteristics of prospective studies

\begin{tabular}{|c|c|c|c|c|}
\hline $\begin{array}{l}\text { Author (year), }{ }^{\text {reference }} \text { study } \\
\text { and year of conduct, } \\
\text { quality score }^{\dagger}\end{array}$ & $\begin{array}{l}\text { Country, study } \\
\text { population }\end{array}$ & $\begin{array}{l}\text { Gender, age } \\
\text { (range or mean } \pm \text { SD) } \\
\text { (years) }\end{array}$ & $\begin{array}{l}\text { Sample } \\
\text { size (cases) }\end{array}$ & $\begin{array}{l}\text { Baseline mean } \pm \text { SD } \\
\text { or } \% 25 \text { OHD, assay }\end{array}$ \\
\hline $\begin{array}{l}\text { Forman et al }(2007),{ }^{22} \\
\text { NHS2 1976-1990, } \\
\text { HPFS 1986-1994, } 5\end{array}$ & $\begin{array}{l}\text { US, nurses and } \\
\text { health professional }\end{array}$ & $34 \%$ male, $43-82$ & $\mathrm{I}, 8 \mathrm{II}(407)$ & $62 \%<75$, RIA \\
\hline $\begin{array}{l}\text { Griffin et al }(201 \mathrm{I}){ }^{23} \\
\text { MBHMS I992-1993, } 6\end{array}$ & $\begin{array}{l}\text { US, general } \\
\text { population }\end{array}$ & I00\% female, $22-44$ & $413(104)$ & Mean $=59,8 I \%<75$, RIA \\
\hline $\begin{array}{l}\text { Anderson et al }(2010),{ }^{24} \\
\text { IHS 2000-2009, } 6\end{array}$ & $\begin{array}{l}\text { US, general } \\
\text { population }\end{array}$ & $25 \%$ male, $55 \pm 21$ & $4 I, 504(2,490)$ & $64 \%<75$, CLIA \\
\hline $\begin{array}{l}\text { Jorde et al }(2010),{ }^{25} \\
\text { Tromsø Study 1994-2008, } 7\end{array}$ & $\begin{array}{l}\text { Norway, general } \\
\text { population }\end{array}$ & $35 \%$ male, $50-74$ & $\mathrm{I}, 268(33 \mathrm{I})$ & $54 \pm 16$, CLIA \\
\hline $\begin{array}{l}\text { Kim et al }(2010),{ }^{26} \\
\text { Chungju city 2003-2007, } 6\end{array}$ & $\begin{array}{l}\text { Korea, general } \\
\text { population }\end{array}$ & $38 \%$ male, $66 \pm 9$ & $\mathrm{I}, 330(85 \mathrm{I})$ & 47, CLIA \\
\hline $\begin{array}{l}\text { Margolis et al }(2012),{ }^{27} \\
\text { WHI I993-1998, } 5\end{array}$ & $\begin{array}{l}\text { US, general } \\
\text { population }\end{array}$ & $100 \%$ female, $50-79$ & $2,153(891)$ & $54 \%<50$, CLIA \\
\hline $\begin{array}{l}\text { Gagnon et al }(2012),{ }^{28} \\
\text { Aus-Diab 2000-2005, } 7\end{array}$ & $\begin{array}{l}\text { Australia, general } \\
\text { population }\end{array}$ & $50 \%$ male, $25-75+$ & $4,164(1,291)$ & 68, RIA \\
\hline $\begin{array}{l}\text { Wang et al }(2013),{ }^{29} \\
\text { PHS 1982-2009, } 5\end{array}$ & US, physicians & $100 \%$ male, $40-84$ & $660(367)$ & $73 \pm 26, \mathrm{RIA}$ \\
\hline $\begin{array}{l}\text { Ke et al }(2013),{ }^{30} \\
\text { ATBC } 1985-1993,4\end{array}$ & $\begin{array}{l}\text { Finland, general } \\
\text { population }\end{array}$ & $\begin{array}{l}100 \% \text { male (smokers), } \\
50-69\end{array}$ & I,957 (252) & $69 \%<50$, RIA \\
\hline $\begin{array}{l}\text { van Ballegooijen et al (20|4), }{ }^{31} \\
\text { MESA 2000-2002, } 5\end{array}$ & $\begin{array}{l}\text { US, general } \\
\text { population }\end{array}$ & $47 \%$ male, $45-84$ & $3,002(1,229)$ & $31 \%<50$, HPLC \\
\hline
\end{tabular}

Note: 'Quality score based on Newcastle-Ottawa Scale.

Abbreviations: NHS2, Nurses' Health Study 2; HPFS, Health Professional Follow-up Study; MBHMS, Michigan Bone Health and Metabolism Study; IHS, Intermountain Healthcare System; WHI, Women's Health Initiative; Aus-Diab, Australian Diabetes, Obesity and Lifestyle Study; PHS, Physicians' Health Study; ATBC, Alpha-Tocopherol, Beta-Carotene Cancer Prevention Study; MESA, Multi-Ethnic Study of Atherosclerosis; SD, standard deviation; 25OHD, 25-hydroxyvitamin D; RR, relative risk; Cl, confidence interval; RIA, radioimmunoassay; US, United States; BMI, body mass index; PA, physical activity; BP, blood pressure; HTN, hypertension; CLIA, chemiluminescence immunoassay.

Risk estimate values of less than 1.00 were associated with a decreased risk for hypertension as a result of increased blood levels of 25OHD. We performed sensitivity analyses to assess the influence of each individual study by removing one study at a time and calculating a pooled estimate for the remainder of the studies. No one study influenced the overall results, suggesting balanced selection.

We tested study heterogeneity by the $R^{2}$ statistic (significance set at $95 \%$ level, ie, $P<0.05) .{ }^{21}$ Potential publication bias was assessed by the Egger's test and presented in a Begg's funnel plot $^{21}$ of standard mean differences against their standard error (Figures 2B and 3B). The meta-analysis procedure was conducted using Comprehensive Meta-Analysis, version 2 .

\section{Results}

\section{Study selection}

Our initial search identified 970 potentially relevant citations (Figure 1). After screening of titles and abstracts, 89 articles remained for further evaluation. Following detailed assessment, 51 articles were excluded. Therefore, 38 studies were included in this study, but only 29 studies (ten prospective studies and 19 cross-sectional studies) were included in the formal meta-analysis; the other nine studies (one prospective study and eight cross-sectional studies) that reported linear results. In addition four studies which were prospective studies and one cross-sectional study, which also reported linear results, are included in Table 5.

\section{Characteristics of included studies - prospective studies}

The pooled RR (95\% CI) for incident hypertension (primary endpoint) in a comparison of individuals in the top category of 25OHD levels with lowest category (as reference group) of 25OHD levels was $0.76(0.63-0.90)$ (Figure 2A).

There was evidence of heterogeneity among the findings of the studies that measured blood 25OHD levels $\left(I^{2}=67.05\right.$, $P<0.01)$. The Egger's test based on all included studies showed no evidence of publication bias for blood 25OHD concentrations and hypertension $(P=0.53)$, with the funnel plot shown in Figure 2B.

Overall, we identified eleven prospective studies, ${ }^{22-32}$ of which ten have been included in this formal meta-analysis. ${ }^{22-31}$ One study by Skaaby et a ${ }^{32}$ from Denmark 


\begin{tabular}{|c|c|c|c|c|}
\hline $\begin{array}{l}250 H D(n m o l / L) \\
\text { comparison }\end{array}$ & $\begin{array}{l}\text { Follow-up } \\
\text { (years) }\end{array}$ & $\begin{array}{l}\text { Primary endpoint: } \\
\text { hypertension } \\
(\mathrm{mmHg})\end{array}$ & $\mathbf{R R}(95 \% \mathrm{Cl})$ & Confounders \\
\hline$<37$ vs $\geqq 75$ & 4 & Self-report & $0.31(0.14-0.72)$ & $\begin{array}{l}\text { Age, BMI, PA, ethnicity, } \\
\text { diabetes status }\end{array}$ \\
\hline$<80$ vs $\geqq 80$ & 14 & $\mathrm{BP} \geqq 140 / 90$ & $0.33(0.12-0.99)$ & $\begin{array}{l}\text { Age, \% body fat, season, HTN } \\
\text { treatment }\end{array}$ \\
\hline$<38$ vs $\geqq 75$ & 1.3 & $\mathrm{BP} \geqq 140 / 90$ & $0.62(0.53-0.73)$ & $\begin{array}{l}\text { Age, gender, season, HTN } \\
\text { treatment, diabetes status }\end{array}$ \\
\hline$<4$ I vs $\geqq 63$ & 14 & $\mathrm{BP} \geqq 140 / 90$ & $0.99(0.76-1.28)$ & $\begin{array}{l}\text { Age, gender, BMI, PA, season, } \\
\text { HTN treatment }\end{array}$ \\
\hline$<20$ vs $\geqq 89$ & 4 & $B P \geqq 140 / 90$ & $0.47(0.27-0.82)$ & $\begin{array}{l}\text { Age, gender, BMI, PA, season, } \\
\text { HTN treatment }\end{array}$ \\
\hline$<34$ vs $\geqq 65$ & 7 & $\mathrm{BP} \geqq 140 / 90$ & $0.86(0.60-1.23)$ & $\begin{array}{l}\text { Age, BMI, PA, ethnicity, season, } \\
\text { HTN treatment, diabetes status }\end{array}$ \\
\hline$<45$ vs $\geqq 85$ & 5 & $\mathrm{BP} \geqq 130 / 85$ & $0.7 \mathrm{I}(0.5 \mathrm{I}-0.98)$ & $\begin{array}{l}\text { Age, gender, PA, ethnicity, } \\
\text { season, diabetes status }\end{array}$ \\
\hline$<50$ vs $\geqq 100$ & 15 & Self-report & $0.94(0.69-1.27)$ & $\begin{array}{l}\text { Age, BMI, PA, ethnicity, season, } \\
\text { HTN treatment, diabetes status }\end{array}$ \\
\hline$<25$ vs $\geqq 80$ & 4 & $\mathrm{BP} \geqq 140 / 90$ & $1.00(0.60-1.50)$ & Age, BMI, PA, season \\
\hline$<50$ vs $\geqq 75$ & 9 & $\mathrm{BP} \geqq 140 / 90$ & $0.8 \mathrm{I}(0.7 \mathrm{I}-0.93)$ & $\begin{array}{l}\text { Age, gender, BMI, PA, ethnicity, } \\
\text { season, HTN treatment }\end{array}$ \\
\hline
\end{tabular}

only reported a linear association between 25OHD and blood pressure and as such, was not included in the formal meta-analysis but is reported in Table 5. In summary, Table 1 presents ten prospective studies with aggregate data on 58,262 nonoverlapping participants and 8,213 incident hypertension cases as potentially relevant for the present meta-analysis.

Thus, Table 1 and Figure 2A include two cohort studies $^{24,25}$ and eight prospective studies, ${ }^{22,23,26-31}$ which were cohort studies in design but only reported baseline 25OHD levels and subsequent future hypertension incidence.

Of these ten studies, two studies ${ }^{25,30}$ also reported baseline as well as follow-up cross-sectional categorical data from the same cohort data, which were included in the cross-sectional meta-analysis (Tables 3 and 4). Four other studies reported baseline $250 \mathrm{HD}$ data in linear format from these cohorts, ${ }^{23,26,27,29}$ and these data are reported in Table 5.

Table 1 provides details of the eligible studies that evaluated vitamin D status and the RRs of hypertension incidence. Six studies ${ }^{22-24,27,29,31}$ were carried out in the US, two studies were conducted in Europe,,$^{25,30}$ and two ${ }^{26,28}$ were conducted in the Asia Pacific region including Australia. ${ }^{28}$ Most of the study samples were from general populations. ${ }^{23-28,30,31}$ In two studies, populations were recruited from nurses, health professionals, or physicians ${ }^{22,29}$ and in one study, the population were male smokers. ${ }^{30}$ The age of participants ranged from 25 years to 85 years (mean age $=55$ years), and more than half of the studies ${ }^{22,24-26,28,31}$ sampled included both male and female. Ascertainment of hypertension was mostly by measuring blood pressure, ${ }^{23-28,30,31}$ and the follow-up period ranged from 1.3 years to 15 years. Table 1 also provides assay characteristics of measured levels of 25OHD from studies contributing to the analyses; five studies ${ }^{22,23,28-30}$ used the radioimmunoassay (RIA) method, four ${ }^{24-27}$ used the chemiluminescence immunoassay (CLIA), and only one ${ }^{31}$ used the gold standard "high-pressure liquid chromatography" (HPLC) analysis.

\section{Vitamin D status and risk of hypertension - prospective studies subgroup analysis}

When studies were stratified by quality score, the RRs were significant for studies rated higher than $5(\mathrm{RR}=0.67$ $(0.51-0.88))$ but were not significant for studies rated $\leqq 5$ 
Table 2 Prospective studies: mixed-effect meta-analysis $250 \mathrm{HD}$ and hypertension stratification

\begin{tabular}{|c|c|c|c|}
\hline Stratification & Sample size & $\begin{array}{l}\text { Risk of hypertension } \\
\text { associated with high } 25 \mathrm{OHD}\end{array}$ & Heterogeneity $\left(I^{2}\right)$ \\
\hline \multicolumn{4}{|l|}{ Journal quality } \\
\hline High (score $=6$ or 7 ) & 48,679 & $0.67(0.5 \mathrm{I}-0.88)^{*}$ & 37.62 \\
\hline Low (score $<6$ points) & 9,583 & $0.86(0.7 I-I .05)$ & $68.67^{*}$ \\
\hline \multicolumn{4}{|l|}{ Demographic } \\
\hline \multicolumn{4}{|l|}{ Age } \\
\hline Older ( $\geqq 55$ years old) & $5 I, 874$ & $0.8 \mathrm{I}(0.67-0.98) *$ & $70.04 *$ \\
\hline Younger ( $<55$ years old) & 6,388 & $0.48(0.26-0.89)^{*}$ & 55.64 \\
\hline \multicolumn{4}{|l|}{ Gender } \\
\hline Both (female and male) & $5 I, 268$ & $0.74(0.59-0.94)^{*}$ & 71.78* \\
\hline Female & 3,764 & $0.55(0.28-1.08)$ & 58.31 \\
\hline Male & 3,230 & $0.88(0.57-1.36)$ & 44.20 \\
\hline \multicolumn{4}{|l|}{ Country region } \\
\hline Asia Pacific & 5,494 & $0.62(0.42-0.90)^{*}$ & 36.50 \\
\hline Europe & 3,225 & $0.99(0.79-1.25)$ & 0.00 \\
\hline US & 49,543 & $0.73(0.57-0.93) *$ & $73.95^{*}$ \\
\hline \multicolumn{4}{|l|}{ Ethnicity } \\
\hline Asian & $\mathrm{I}, 330$ & $0.47(0.27-0.82)^{*}$ & 0.00 \\
\hline Caucasian & 53,930 & $0.76(0.6 \mathrm{I}-0.94)^{*}$ & $65.99 *$ \\
\hline Multi-ethnic groups (US) & 3,002 & $0.89(0.76-1.05)$ & 0.00 \\
\hline \multicolumn{4}{|l|}{ Vitamin D } \\
\hline \multicolumn{4}{|l|}{ Vitamin D levels } \\
\hline High ( $\geqq 50 \mathrm{nmol} / \mathrm{L})$ & 53,707 & $0.73(0.59-0.90) *$ & $68.90 *$ \\
\hline Low $(<50 \mathrm{nmol} / \mathrm{L})$ & 4,555 & $0.81(0.53-1.24)$ & 66.24 \\
\hline \multicolumn{4}{|l|}{ High age, low $25 \mathrm{OHD}$} \\
\hline No & 53,707 & $0.73(0.59-0.90) *$ & $68.90 *$ \\
\hline Yes & 4,555 & $0.81(0.53-1.24)$ & 66.24 \\
\hline \multicolumn{4}{|l|}{ Season } \\
\hline No season & $\mathrm{I}, 8 \mathrm{II}$ & $0.31(0.14-0.72)^{*}$ & 0.00 \\
\hline Seasons & 56,451 & $0.78(0.66-0.93)^{*}$ & $64.88^{*}$ \\
\hline \multicolumn{4}{|l|}{ Assay method } \\
\hline CLIA & 46,255 & $0.73(0.54-0.99)^{*}$ & $75.78^{*}$ \\
\hline HPLC & 3,002 & $0.89(0.76-1.05)$ & 0.00 \\
\hline RIA & 9,005 & $0.7 \mid(0.50-1.00)$ & $59.06 *$ \\
\hline \multicolumn{4}{|l|}{ Hypertension } \\
\hline \multicolumn{4}{|l|}{ Hypertension self-report } \\
\hline No & 55,791 & $0.76(0.63-0.92) *$ & $66.90 *$ \\
\hline Yes & $2,47 \mid$ & $0.58(0.20-1.69)$ & $83.11 *$ \\
\hline \multicolumn{4}{|l|}{ Antihypertensive treatment } \\
\hline No & 49,436 & $0.66(0.5 \mathrm{I}-0.87)^{*}$ & 54.57 \\
\hline Yes & 8,826 & $0.85(0.70-1.01)$ & 44.52 \\
\hline \multicolumn{4}{|l|}{ Confounders } \\
\hline \multicolumn{4}{|l|}{ BMI } \\
\hline No & 48,670 & $0.63(0.55-0.73)^{*}$ & 0.00 \\
\hline Yes & 9,592 & $0.8 \mathrm{I}(0.66-0.98) *$ & $53.78^{*}$ \\
\hline \multicolumn{4}{|l|}{ PA } \\
\hline No & 41,917 & $0.58(0.39-0.84)^{*}$ & 19.00 \\
\hline Yes & 16,345 & $0.82(0.69-0.97)^{*}$ & 48.15 \\
\hline \multicolumn{4}{|l|}{ Diabetes } \\
\hline No & 7,970 & $0.82(0.63-1.05)$ & 55.94 \\
\hline Yes & 50,292 & $0.71(0.56-0.90) *$ & $63.02 *$ \\
\hline
\end{tabular}

Note: *Significance $P<0.05$.

Abbreviations: 25OHD, 25-hydroxyvitamin D; US, United States; CLIA, chemiluminescence immunoassay; HPLC, high-pressure liquid chromatography; RIA, radioimmunoassay; BMI, body mass index; PA, physical activity.

$(\mathrm{RR}=0.86(0.71-1.05))$. There was no statistically significant heterogeneity among studies rated higher than $5\left(I^{2}=37.62, P>0.05\right)$, but significant heterogeneity was found among studies rated $\leqq 5\left(I^{2}=68.67, P<0.05\right)$. Detailed results when studies were stratified by demographic factors (age, gender, country region, and ethnicity), vitamin D factors (vitamin D levels, season, assay methods), hypertension (measured or self-report, history of anti-hypertensive use), and confounders (BMI, PA, and diabetes status) are given in Table 2 and Figure 2A-D. 


\section{Characteristics of included studies - cross-sectional studies}

The pooled OR-estimated risk of hypertension (primary endpoint) for the highest vs the lowest category of blood $25 \mathrm{OHD}$ concentrations was $0.79(0.73-0.87)$ (Figure 3A). There was statistically significant heterogeneity among all crosssectional studies $\left(P^{2}=45.37, P<0.05\right)$. The Egger's test based on all included studies showed no evidence of publication bias for blood 25OHD concentrations and hypertension $(P=0.62)$, together with the funnel plot shown in Figure 3B.

Twenty-seven cross-sectional studies were identified, of which $19^{25,30,33-49}$ were included in this formal meta-analysis (Tables 3 and 4 and Figure 3A). Of these 19 cross-sectional studies, one study ${ }^{48}$ also reported linear results that are reported in Table 5. The remaining extracted cross-sectional studies only reported 25OHD estimates in linear fashion and thus were not included in the formal meta-analysis but are reported in Table 5. ${ }^{29,48,50-57}$

In summary, Tables 3 and 4 represent 19 crosssectional studies with aggregate data on 90,535 nonoverlapping participants. Tables 3 and 4 provide details of the eligible studies that evaluated vitamin D status and the ORs of hypertension. Six studies were carried out in the US, ${ }^{33,34,36,41,43,44}$ five studies in Europe, , $5,30,35,37,40$ five studies in the Asia Pacific ${ }^{38,39,46-48}$ (including one in Australia ${ }^{39}$ ), one in Middle-East, ${ }^{42}$ one in Puerto Rico, ${ }^{45}$ and one in South Africa. ${ }^{49}$ Sixteen studies were recruited from the general population,,$^{37-44,46,48,49}$ and two studies were sampled from either clinic attendees ${ }^{45}$ or factory employees. ${ }^{47}$ The age of participants ranged from 18 years to 92 years, and only five studies were of one gender, ${ }^{30,36,39,40,49}$ and the majority were mixed. Ascertainment of hypertension was mostly by measuring blood pressure and in only two studies, ${ }^{36,44}$ it was from self-report.

Tables 3 and 4 also provides assay characteristics of measured levels of 25OHD from studies contributing to the analyses. Nine cross-sectional studies ${ }^{30,33,38,39,41,42,44,47,48}$ used the RIA method, six cross-sectional studies used CLIA or electro-chemiluminescence immunoassay, ${ }^{25,34-36,45,46}$ one used enzyme-linked immunosorbent assay, ${ }^{37}$ one used Nichols Advantage, ${ }^{43}$ one used Roche Elecsys, ${ }^{49}$ and one used HPLC ${ }^{40}$ for blood 25OHD analyses.

\section{Vitamin D status and risk of hypertension - cross-sectional studies subgroup analysis}

When studies were stratified by quality score, the ORs were significant for studies rated $5(\mathrm{OR}=0.71(0.61-0.83))$ and $6(\mathrm{OR}=0.79(0.73-0.87))$ but were not significant for studies rated $\leqq 4(\mathrm{OR}=0.87(0.75-1.01))$. There was no statistically significant heterogeneity among studies rated at $4\left(I^{2}=46.13, P>0.05\right), 5\left(I^{2}=39.34, P>0.05\right)$, or $6\left(I^{2}=0.00, P>0.05\right)$. Detailed results when studies were stratified by demographic factors (age, gender, country region, and ethnicity), vitamin D factors (vitamin D levels, seasons, and assay methods), hypertension (measured or self-report, history of anti-hypertensive use), and confounders (BMI, PA, and diabetes status) are given in Table 4 and Figure 3A-D.

\section{Discussion}

\section{Physiology of vitamin D}

In humans, vitamin D is normally obtained from skin through the action of ultraviolet $\mathrm{B}$ irradiation on 7-dehydrocholesterol. ${ }^{58}$ It is further metabolized to $25 \mathrm{OHD}$, the major circulating vitamin D compound, and then to 1,25-dihydroxyvitamin $\mathrm{D}(1,25 \mathrm{D})$, the hormonal form. ${ }^{46,9}$ The major function of vitamin D compounds is to enhance active absorption of ingested calcium (and phosphate). This assists in building bone at younger ages and ensures that bone does not need to be resorbed to maintain blood calcium concentrations. As there are vitamin D receptors in most nucleated cells, including vascular smooth muscle cells, as well as in macula densa and juxtaglomerular cells, ${ }^{59,60}$ vitamin D compounds appear to have direct effects to improve bone and muscle function, and there is good, though not entirely consistent, evidence that supplemental vitamin D and calcium together reduce falls and fractures in older individuals. ${ }^{61}$ Based on calcium control and musculoskeletal function, target levels of 25OHD in blood are at least $50-60 \mathrm{nmol} / \mathrm{L}$, and there may be a case for higher targets of $75-80 \mathrm{nmol} / \mathrm{L}$. ${ }^{10,11}$

\section{Summary and comparison with other meta-analyses}

The overall results from this recent meta-analysis and systematic review are that from a total of 148,797 healthy participants $(58,262$ prospective; 90,535 cross-sectional) from general population samples across the world (published between 2007 and early 2014), lower 25OHD levels appear to be associated with increased hypertension levels (prospective analyses: $\mathrm{RR}=0.76(0.63-0.90)$; crosssectional analyses: $\mathrm{OR}=0.79(0.73-0.87))$. These updated results report similar findings published by Kunutsor et al for prospective studies in $2013(\mathrm{RR}=0.70(0.58-0.86))^{8}$ and for cross-sectional studies by Burgaz et al in 2011 $(\mathrm{OR}=0.73(0.63-0.84))^{7}$ 
Table 3 Characteristics of cross-sectional studies

\begin{tabular}{|c|c|c|c|}
\hline $\begin{array}{l}\text { Author (year), reference study and year } \\
\text { of conduct, quality score }\end{array}$ & Country, study sample & $\begin{array}{l}\text { Gender, age range or } \\
\text { mean } \pm \text { SD (years) }\end{array}$ & Sample size, assay \\
\hline Martins et al (2007), ${ }^{33}$ NHANES I988-1994, 6 & US, general population & $48 \%$ male, $20-80$ & I5,088, RIA \\
\hline Reis et al (2007), ${ }^{34}$ RBS 1997-1999, 5 & US, general population & $38 \%$ male, $44-96$ & $\mathrm{I}, 070, \mathrm{CLIA}$ \\
\hline Snijder et al (2007), ${ }^{35}$ LASA 1996, 6 & $\begin{array}{l}\text { The Netherlands, general } \\
\text { population }\end{array}$ & $50 \%$ male, $55-85$ & I,205, CLIA \\
\hline Forman et al (2008), ${ }^{36}$ NHS2 1997-1999, 4 & US, nurses & $100 \%$ female, $32-52$ & I,484, CLIA \\
\hline Hypponen et al (2008), ${ }^{37}$ I958 BBC 2002-2004, 5 & UK, general population & $48 \%$ male, $45-47$ & 6,293, ELISA \\
\hline Lu et al (2009), ${ }^{38}$ NHAPC, 2005, 5 & $\begin{array}{l}\text { People's Republic of China, } \\
\text { general population }\end{array}$ & $44 \%$ male, $50-70$ & 3,262, RIA \\
\hline Pasco et al (2009), ${ }^{39}$ GOS 1994-1997, 5 & Australia, general population & $100 \%$ female, $20-92$ & $86 I$, RIA \\
\hline Burgaz et al $(2010),,^{40}$ ULSAM I99I-1995, 6 & Sweden, general population & $100 \%$ male, $7 I \pm 0.6$ & 830, HPLC/MS \\
\hline Jorde et al (2010), ${ }^{25}$ Tromsø Study 2008, 4 & Norway, general population & $37 \%$ male, $25-84$ & 4,I25, ECLIA \\
\hline Zhao et al $(2010),{ }^{41}$ NHANES 2003-2006, 6 & US, general population & $49 \%$ male, $\geqq 20$ & $7,228, \mathrm{RIA}$ \\
\hline Steinvil et al $(20 \mathrm{II}), 42$ Maccabi Healthcare & Israel, general population & $23 \%$ male, $55 \pm 17$ & $34,874, \mathrm{RIA}$ \\
\hline Services $200 \mathrm{I}-2008,4$ & & $77 \%$ female, $55 \pm 15$ & \\
\hline Bhandari et al (20I I), ${ }^{43}$ KPSCHP 2004-2006, 5 & US, general population & $31 \%$ male, $>59$ & 2,722, Nichols Advantage \\
\hline Brock et al (20II), ${ }^{44}$ PLCO 1993-200I, 4 & US, general population & $53 \%$ male, $55-74$ & 2,465, RIA \\
\hline Caro et al $(2012), 45$ no name and year, 4 & Puerto Rico, clinic subjects & $15 \%$ male, $21-50$ & 219, CLIA \\
\hline $\begin{array}{l}\text { Dorjgochoo et al (2012), }{ }^{46} \text { SWHS 1997-2000, } \\
\text { SMHS 2002-2006, } 4\end{array}$ & $\begin{array}{l}\text { People's Republic of China, } \\
\text { general population }\end{array}$ & $28 \%$ male, $40-75$ & I,460, CLIA \\
\hline Sumriddetchkajorn et al (2012), ${ }^{47}$ no name 1997, 4 & Thailand, factory employees & $14 \%$ male, $35-54$ & 274, RIA \\
\hline Kim et al $(2013),{ }^{48}$ KNHANES 2009-2010, 5 & Korea, general population & $45 \%$ male, $50-90$ & $4,5 \mid 3, \mathrm{RIA}$ \\
\hline Kruger et al (2013), ${ }^{49}$ PURE 2005, 4 & $\begin{array}{l}\text { South Africa, general } \\
\text { population }\end{array}$ & $100 \%$ female, $58 \pm 9$ & 291, Roche Elecsys \\
\hline Ke et al $(2013),{ }^{30}$ ATBC 1985-1993, 4 & Finland, general population & $100 \%$ male (smoker), $50-69$ & $2,27 I, R I A$ \\
\hline
\end{tabular}

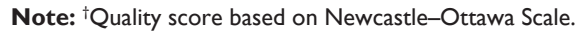

Abbreviations: NHANES, National Health and Nutrition Examination Survey; RBS, Rancho Bernardo Study; LASA, Longitudinal Aging Study Amsterdam; NHS2, Nurses' Health Study 2; BBC, British Birth Cohort; NHAPC, Nutrition and Health of Aging Population in China; GOS, Geelong Osteoporosis Study; ULSAM, Uppsala Longitudinal Study of Adult Men; KPSCHP, Kaiser Permanente Southern California Health Plan; PLCO, Prostate, Lung, Colorectal and Ovarian Cancer Screening Trial; SWHS, Shanghai Women's Health Study; SMHS, Shanghai Men's Health Study; KNHANES, Korea National Health and Nutrition Examination Survey; PURE, Prospective Urban Rural Epidemiology Study; ATBC, Alpha-Tocopherol, Beta-Carotene Cancer Prevention Study; SD, standard deviation; 25OHD, 25-hydroxyvitamin D; OR, odds ratio; Cl, confidence interval; RIA, radioimmunoassay; US, United States; UK, United Kingdom; BP, blood pressure; BMI, body mass index; HTN, hypertension; CLIA, chemiluminescence immunoassay; PA, physical activity; WC, waist circumference; ELISA, enzyme-linked immunosorbent assay; HPLC, high-pressure liquid chromatography; MS, mass spectrometry; ECLIA, electro-chemiluminescence immunoassay; SBP, systolic blood pressure.

In addition, our systematic review of 172,259 participants and 38 studies concurs with observations from the above meta-analyses in that $60 \%$ of studies reported a significant inverse association between blood 25OHD and hypertension. Of these, $40 \%$ were reported from prospective studies (Table 1), $56 \%$ from cross-sectional studies (Table 3 ), and $64 \%$ from those studies that reported linear associations of $25 \mathrm{OHD}$ with hypertension (Table 5).

It should be noted that mostly these effects were of a small magnitude and have not been confirmed in results from RCTs. ${ }^{14}$ Probably, because of these facts and other even less-convincing data from mortality studies ${ }^{62,63}$ and other CVD outcomes, ${ }^{4-6,9}$ critics have hypothesized that observational associations may be due to an association between low vitamin D and general "ill health", ${ }^{64}$ a conclusion observational studies cannot fully address due to their study design. Thus, the major criticism of observational studies is that results may be due to reverse causality (such as already unwell participants) and/or unmeasured bias or confounding (such as obesity and lack of PA), which RCTs attempt to take into account by randomization. This review has attempted to address 


\begin{tabular}{|c|c|c|c|c|}
\hline $\begin{array}{l}\text { Mean or } \% \\
250 H D(n m o l / L)\end{array}$ & $\begin{array}{l}250 H D(n m o l / L) \\
\text { comparison }\end{array}$ & $\begin{array}{l}\text { Primary endpoint: } \\
\text { hypertension }(\mathrm{mmHg})\end{array}$ & OR $(95 \% \mathrm{Cl})$ & Confounders \\
\hline $75,60 \%<75$ & $<53$ vs $\geqq 93$ & $\mathrm{BP} \geqq 140 / 90$ & $0.78(0.67-0.88)$ & $\begin{array}{l}\text { Age, gender, BMI, ethnicity, season, } \\
\text { HTN treatment }\end{array}$ \\
\hline Male: 109 & $<78$ vs $\geqq 120$ & $\mathrm{BP} \geqq 130 / 85$ & $1.28(0.58-2.82)$ & Age, abdominal obesity, PA, season, \\
\hline Female: 102 & $<88$ vs $\geqq 126$ & & $1.01(0.53-1.93)$ & HTN treatment, diabetes status \\
\hline $37 \%<50$ & $<25$ vs $\geqq 75$ & $\mathrm{BP} \geqq 140 / 90$ & $1.12(0.59-2.13)$ & $\begin{array}{l}\text { Age, gender, BMI, WC, PA, season, } \\
\text { HTN treatment }\end{array}$ \\
\hline $68,66 \%<75$ & $<42$ vs $\geqq 95$ & Self-reported & $0.60(0.40-0.90)$ & Age, BMI, PA, ethnicity, season \\
\hline $\begin{array}{l}\text { Male: } 54 \text {, } \\
\text { Female: } 52\end{array}$ & $<27$ vs $\geqq 150$ & $B P \geqq 140 / 90$ & $0.72(0.61-0.85)$ & $\begin{array}{l}\text { Age, gender, BMI, PA, season, HTN } \\
\text { treatment }\end{array}$ \\
\hline $40,70 \%<50$ & $<29$ vs $\geqq 58$ & $\mathrm{BP} \geqq 130 / 85$ & $0.66(0.51-0.85)$ & $\begin{array}{l}\text { Age, gender, BMI, PA, season, HTN } \\
\text { treatment, diabetes status }\end{array}$ \\
\hline $33 \%<50$ & $<53$ vs $\geqq 74$ & $\mathrm{BP} \geqq 140 / 90$ & $0.40(0.22-0.70)$ & $\begin{array}{l}\text { Age, weight, season, HTN } \\
\text { treatment }\end{array}$ \\
\hline 70 & $<38$ vs $\geqq 50-75$ & $\mathrm{BP} \geqq 140 / 90$ & $0.31(0.09-1.11)$ & $\begin{array}{l}\text { Age, BMI, PA, season, HTN } \\
\text { treatment }\end{array}$ \\
\hline 54 & $<4 \mid$ vs $\geqq 63$ & $\mathrm{BP} \geqq 160 / 95$ & $0.87(0.60-1.27)$ & $\begin{array}{l}\text { Age, gender, BMI, PA, HTN } \\
\text { treatment }\end{array}$ \\
\hline 80 & $<38$ vs $\geqq 75$ & $\mathrm{BP} \geqq 140 / 90$ & $0.80(0.72-0.89)$ & $\begin{array}{l}\text { Age, gender, BMI, PA, ethnicity, } \\
\text { HTN treatment, diabetes status }\end{array}$ \\
\hline $79 \%$ male $<75$ & $<38$ vs $\geqq 75$ & $\mathrm{BP} \geqq 160 / 95$ & $0.90(0.77-1.05)$ & Age, HTN treatment \\
\hline $78 \%$ female $<75$ & & & $0.84(0.76-0.92)$ & \\
\hline $15 \%<75$ & $<37$ vs $\geqq 100$ & $\mathrm{BP} \geqq 160 / 95$ & $0.37(0.19-0.88)$ & $\begin{array}{l}\text { Age, gender, ethnicity, HTN } \\
\text { treatment }\end{array}$ \\
\hline $29 \%<50$ & $<37$ vs $\geqq 80$ & Self-report & $1.00(0.70-1.40)$ & Age, gender, BMI, PA, season \\
\hline $60 \%<75$ & $<75$ vs $\geqq 75$ & $\mathrm{BP} \geqq 140 / 90$ & $0.90(0.29-2.86)$ & Age, gender, BMI, HTN treatment \\
\hline $96 \%<75$ & $<24$ vs $\geqq 5$ I & $\mathrm{BP} \geqq 140 / 90$ & $0.86(0.38-1.95)$ & $\begin{array}{l}\text { Age, gender, BMI, PA, season, HTN } \\
\text { treatment }\end{array}$ \\
\hline $36 \%<70$ & $<70$ vs $\geqq 70$ & $\mathrm{BP} \geqq 140 / 90$ & $1.82(1.06-3.03)$ & Age, gender, BMI \\
\hline $49,53 \%<50$ & $<25$ vs $\geqq 50$ & $\mathrm{BP} \geqq 140 / 90$ & $\begin{array}{l}0.80(0.61-1.05) \\
0.76(0.59-0.98)\end{array}$ & Age, BMI, PA, HTN treatment, \\
\hline $63 \%<75$ & $<75$ vs $\geqq 75$ & $\mathrm{SBP} \geqq 146$ & $0.54(0.31-0.95)$ & Age, BMI \\
\hline $69 \%<50$ & $<25$ vs $\geqq 80$ & $\mathrm{BP} \geqq 140 / 90$ & $0.90(0.60-1.40)$ & Age, BMI, season \\
\hline
\end{tabular}

some of these issues by stratification and sensitivity analyses.

\section{Quality of included studies}

When prospective studies were stratified by the journal quality ratings score ( $\geq 6$ vs lower), ${ }^{19}$ the association between low $25 \mathrm{OHD}$ and increased hypertension was both significant and not heterogeneous in the better-quality studies ${ }^{23-26,28}$ (score $\geq 6, \mathrm{RR}=0.67(0.51-0.88)$; score $<6, \mathrm{RR}=0.86$ (0.71-1.05)) (Figure 2C). A similar pattern occurred in cross-sectional studies, although the effect was not quite as marked in that all the studies remained significant in their category and also had no heterogeneity, but the "higher quality" ${ }^{33,35,40,41}$ studies showed a stronger association between lower 25OHD levels and hypertension vs "moderate quality" $34,37-39,43,48$ (score $=6$, OR $=0.79(0.73-$ $0.87)$; score $=5, \mathrm{OR}=0.72(0.65-0.80)$; score $=4, \mathrm{OR}=0.86$ $(0.80-0.93))$ (Figure 3C).

\section{Heterogeneity and publication bias}

As with the previous meta-analyses, there was no publication bias reported, probably due to the strict criteria used for selecting the studies to be used. However, in contrast to the two previous meta-analysis, ${ }^{7.8}$ which reported no heterogeneity in their results, this present meta-analysis reported heterogeneity among studies of blood 25OHD concentrations and 
Table 4 Cross-sectional studies: mixed-effect meta-analysis $25 \mathrm{OHD}$ and hypertension stratification

\begin{tabular}{|c|c|c|c|}
\hline Stratification & Sample size & $\begin{array}{l}\text { Risk of hypertension } \\
\text { associated with high } 25 \mathrm{OHD}\end{array}$ & Heterogeneity $\left(I^{2}\right)$ \\
\hline Total & 90,535 & $0.79(0.73-0.87)^{*}$ & $45.37 *$ \\
\hline \multicolumn{4}{|l|}{ Journal quality } \\
\hline High (score =6) & 23,521 & $0.79(0.73-0.87)^{*}$ & 0.00 \\
\hline Middle (score =5) & 23,676 & $0.7 \mathrm{I}(0.6 \mathrm{I}-0.83)^{*}$ & 39.43 \\
\hline Low $($ score $=4)$ & 43,338 & $0.87(0.75-1.01)$ & 46.13 \\
\hline \multicolumn{4}{|l|}{ Demographic } \\
\hline \multicolumn{4}{|l|}{ Age } \\
\hline Older ( $\geqq 55$ years old) & 18,736 & $0.79(0.70-0.89)^{*}$ & 0.00 \\
\hline Younger ( $<55$ years old) & 71,799 & $0.79(0.70-0.88)^{*}$ & $63.83 *$ \\
\hline \multicolumn{4}{|l|}{ Gender } \\
\hline Both (female and male) & 42,881 & $0.8 \mathrm{I}(0.7 \mathrm{I}-0.93)^{*}$ & $57.76^{*}$ \\
\hline Female & 33,540 & $0.72(0.59-0.87)^{*}$ & 45.96 \\
\hline Male & $14,1 \mid 4$ & $0.86(0.75-0.99)^{*}$ & 6.68 \\
\hline \multicolumn{4}{|l|}{ Country region } \\
\hline Asia Pacific & 10,370 & $0.78(0.59-1.04)$ & $70.21^{*}$ \\
\hline Europe & 14,724 & $0.79(0.65-0.95)^{*}$ & 17.93 \\
\hline US & 35,384 & $0.79(0.68-0.91)^{*}$ & 3.39 \\
\hline Others & 30,057 & $0.85(0.78-0.93)^{*}$ & 44.10 \\
\hline \multicolumn{4}{|l|}{ Ethnicity } \\
\hline Asian & 9,509 & $0.85(0.65-1.12)$ & $66.10^{*}$ \\
\hline Caucasian & 20,604 & $0.79(0.55-0.95) *$ & 42.63 \\
\hline Multi-ethnic groups (US) & 17,810 & $0.58(0.28-1.18)$ & $78.86^{*}$ \\
\hline Others & 42,612 & $0.83(0.78-0.89)^{*}$ & 0.00 \\
\hline \multicolumn{4}{|l|}{ Vitamin D } \\
\hline \multicolumn{4}{|l|}{ Vitamin D level } \\
\hline High ( $\geqq 50$ nmol/L) & 76,408 & $0.78(0.7 \mathrm{I}-0.86)^{*}$ & $46.98^{*}$ \\
\hline Low $(<50 \mathrm{nmol} / \mathrm{L})$ & 14,127 & $0.85(0.70,1.05)$ & 49.38 \\
\hline \multicolumn{4}{|l|}{ High age, low $25 \mathrm{OHD}$} \\
\hline No & 78,166 & $0.79(0.7 \mathrm{I}-0.88)^{*}$ & $57.63^{*}$ \\
\hline Yes & 12,369 & $0.81(0.70-0.94)^{*}$ & 0.00 \\
\hline \multicolumn{4}{|l|}{ Season } \\
\hline No season & 55,107 & $0.80(0.7 \mid-0.9 I)^{*}$ & $56.60^{*}$ \\
\hline Season & 35,428 & $0.77(0.69-0.86)^{*}$ & 23.45 \\
\hline \multicolumn{4}{|l|}{ Assay method } \\
\hline CLIA & 9,563 & $0.84(0.68-1.05)$ & 0.00 \\
\hline ELISA & 6,293 & $0.72(0.6 \mathrm{I}-0.85)^{*}$ & 0.00 \\
\hline HPLC & 830 & $0.31(0.09-1.09)$ & 0.00 \\
\hline Nichols Advantage & 2,722 & $0.37(0.19-0.71)^{*}$ & 0.00 \\
\hline RIA & 70,836 & $0.82(0.78-0.87)^{*}$ & $53.90 *$ \\
\hline Roche & 291 & $0.54(0.3 \mathrm{I}-0.95)^{*}$ & 0.00 \\
\hline \multicolumn{4}{|l|}{ Hypertension } \\
\hline \multicolumn{4}{|l|}{ Hypertension self-report } \\
\hline No & 86,586 & $0.79(0.73-0.87)^{*}$ & $45.6 I^{*}$ \\
\hline Yes & 3,949 & $0.78(0.48-1.29)$ & $71.50 *$ \\
\hline \multicolumn{4}{|l|}{ Antihypertensive treatment } \\
\hline No & 6,785 & $0.88(0.6 \mathrm{I}-\mathrm{I} .28)$ & $71.95^{*}$ \\
\hline Yes & 83,750 & $0.79(0.73-0.85)^{*}$ & 31.37 \\
\hline \multicolumn{4}{|l|}{ Confounders } \\
\hline \multicolumn{4}{|l|}{ BMI } \\
\hline No & 37,596 & $0.81(0.66-0.99) *$ & $70.32 *$ \\
\hline Yes & 52,939 & $0.79(0.7 \mathrm{I}-0.87)^{*}$ & $39.00 *$ \\
\hline \multicolumn{4}{|l|}{ PA } \\
\hline No & 56,600 & $0.79(0.67-0.94)^{*}$ & $67.7 I^{*}$ \\
\hline Yes & 33,935 & $0.78(0.72-0.84)^{*}$ & 1.63 \\
\hline \multicolumn{4}{|l|}{ Diabetes status } \\
\hline No & 74,462 & $0.80(0.70-0.90) *$ & $55.87^{*}$ \\
\hline Yes & 16,073 & $0.79(0.72-0.86)^{*}$ & 0.00 \\
\hline
\end{tabular}

Note: *Significance $P<0.05$.

Abbreviations: 25OHD, 25-hydroxyvitamin D; US, United States; CLIA, chemiluminescence immunoassay; ELISA, enzyme-linked immunosorbent assay; HPLC, highpressure liquid chromatography; RIA, radioimmunoassay; BMI, body mass index; PA, physical activity. 
hypertension (in both prospective and cross-sectional studies) (Tables 2 and 4). The previous meta-analyses restricted their study selection to defined populations (eg, primarily Caucasian from either Europe or the US). The heterogeneity reported in the present meta-analysis is probably due to the wide scope of the literature search in demographic range (age, gender, country location, and ethnicity) of the studies assessed and also the different 25OHD assay technologies used.

A

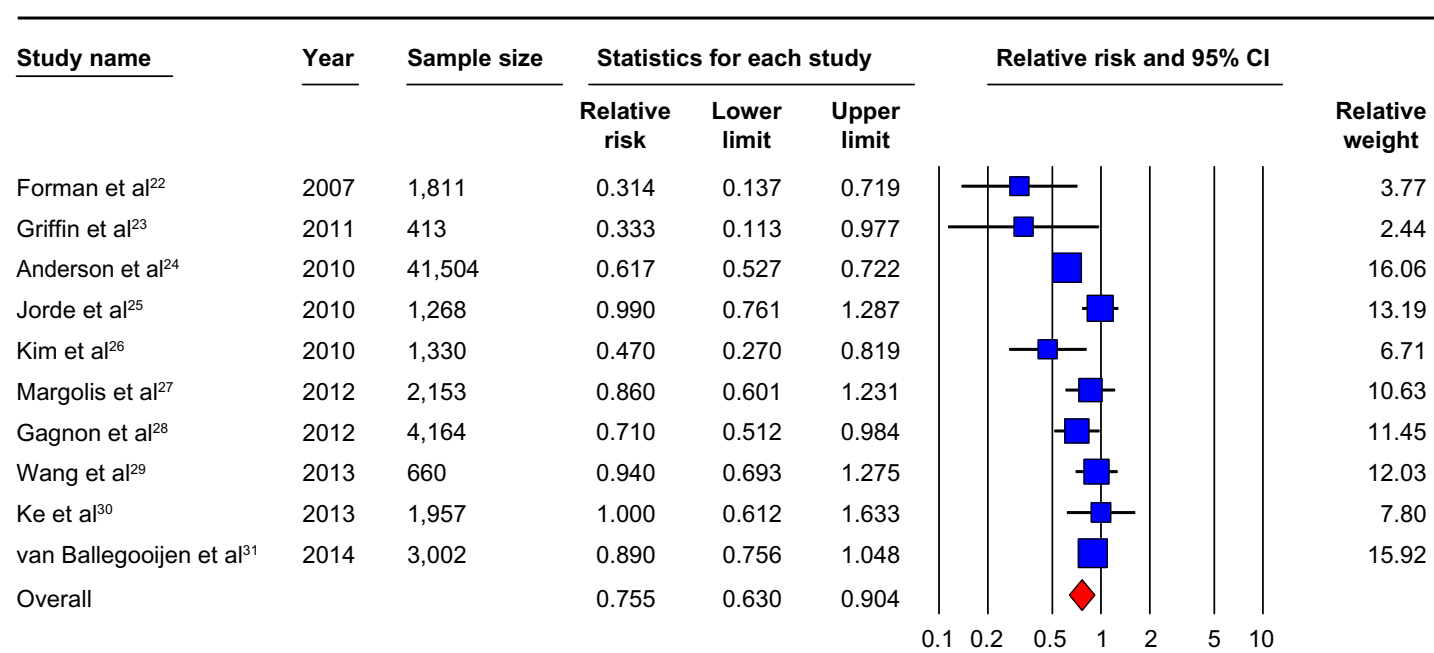

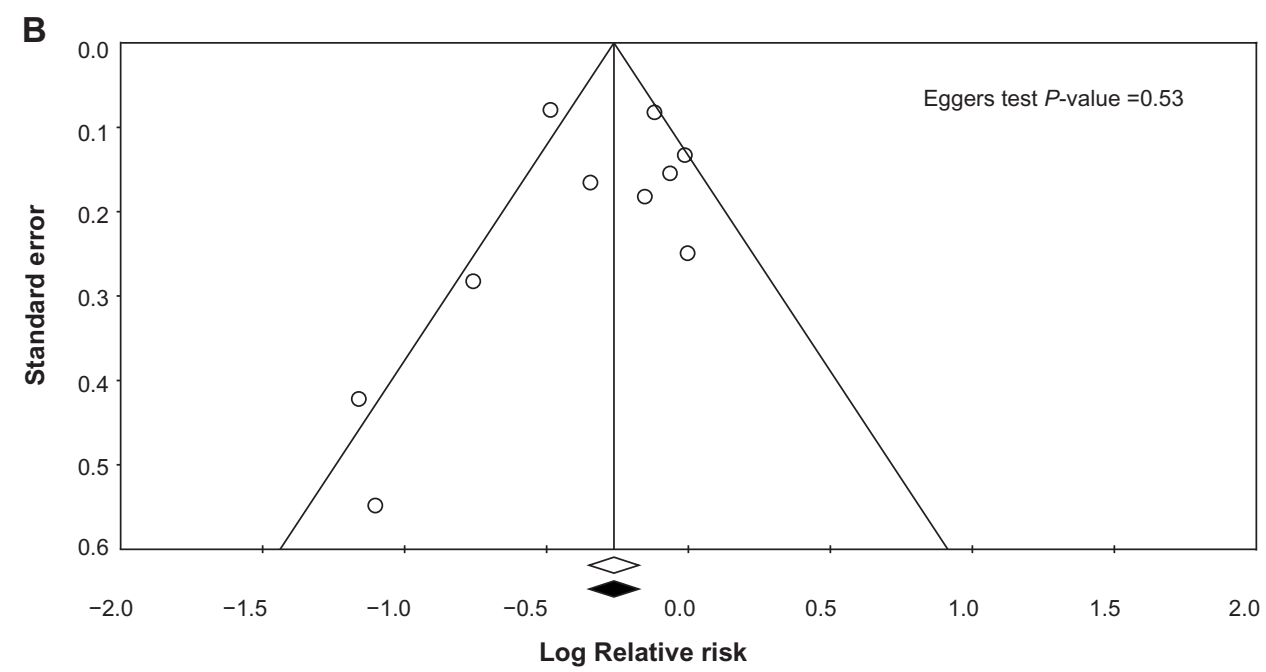

C

Quality score Study name

$<6$
$<6$
$<6$
$<6$
$<6$
$<6$
$\geq 6$
$\geq 6$
$\geq 6$
$\geq 6$
$\geq 6$
$\geq 6$
Overall

Figure 2 (Continued)

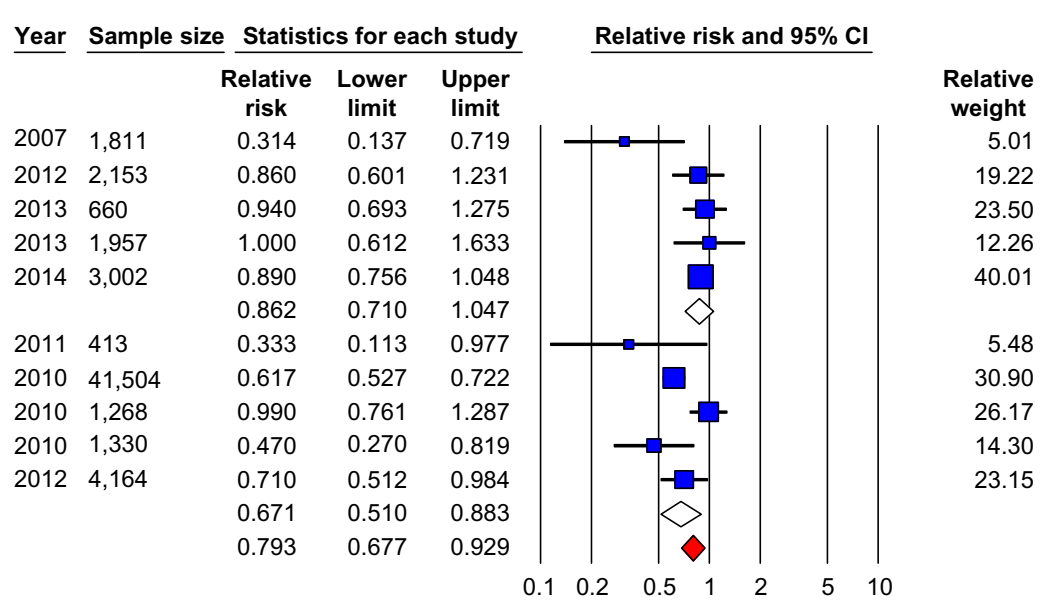




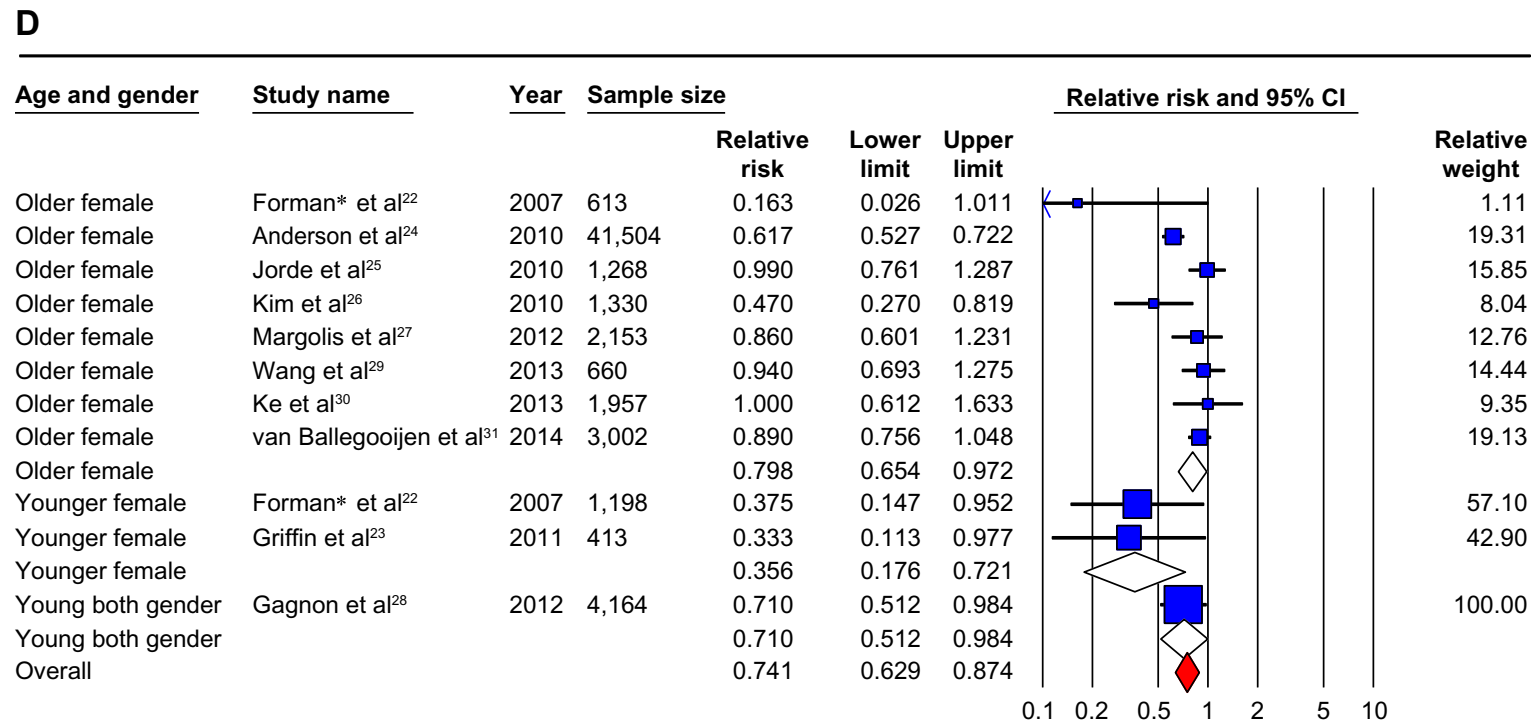

Figure 2 Prospective studies.

Notes: (A) Prospective studies of vitamin D and hypertension risk: the effect of higher vitamin D (measured as quartiles of $25 \mathrm{OHD}$ ) on hypertension risk expressed as relative risk (RR) for individual studies (blue) and the calculated overall RR (red). (B) Funnel plot showing standard error by log RR for the prospective studies. (C) Prospective studies of vitamin $D$ and hypertension risk sub-groups: the effect of higher vitamin $D$ (measured as quartiles of $25 \mathrm{OHD}$ ) on hypertension risk stratified by quality score expressed as RR for individual studies (blue), sub-groups total (white) and the calculated overall RR (red). (D) Prospective studies of vitamin D and hypertension risk sub-groups: the effect of higher vitamin D (measured as quartiles of 25OHD) on hypertension risk stratified by age and gender expressed as RR for individual studies (blue), sub-group total (white) and the calculated overall RR (red). *Studies published in one paper.

Abbreviation: $\mathrm{Cl}$, confidence Interval.

\section{Demography and ethnicity}

The age range in this review was 18-96, and the effect of low 25OHD on hypertension remained significant in both younger and older strata and somewhat surprisingly, was markedly stronger in those aged $<55$, and in females in prospective and cross-sectional data (Figures 2D and 3D).

When it was possible to investigate gender separately, heterogeneity disappeared in both prospective and cross-sectional studies, but the associations became nonsignificant in the prospective studies, probably due to lower sample sizes. Females did seem to have a greater degree of association of hypertension risk with low vitamin $\mathrm{D}$, especially at a younger age. This is puzzling as one would not expect younger females to have increased risk of hypertension, but increasingly younger females are being reported to be at risk for vitamin D deficiency, particularly in Asian populations. ${ }^{65}$ These findings need to be further investigated; our recent data from Macau also confirm this risk in younger women. ${ }^{66,67}$

When investigated by region or country of the study population, countries from Europe and the US were more similar (ie, less heterogeneity) than those from other regions of the world; previous meta-analyses have concentrated on these countries. In this meta-analysis, the effect of vitamin D deficiency on hypertension was attenuated in the European studies compared to the total risk estimate and was, in fact, non-significant from prospective data. When ethnicity was investigated, those of "multi-ethnic group" ethnicities did not show an effect compared with others; as Burgaz et $\mathrm{al}^{7}$ noted, there is evidence that vitamin D synthesis is less efficient among individuals with greater skin pigmentation, ${ }^{68,69}$ but variations in culturally preferred covering of skin by clothing could also be an explanation for these findings. ${ }^{70}$ More studies in different cultures are needed to disentangle these findings.

\section{Measurement of blood pressure variables}

Only three studies included in the present meta-analysis have used self-reported hypertension as outcomes (two in prospective ${ }^{22,27}$ and one in cross-sectional ${ }^{44}$ ), which can only give a crude estimate of average blood pressure. In our stratified analysis, the effect was attenuated when studies took account of measured blood pressure, but the negative association between decreased 25OHD and hypertension remained significant. In addition, taking into account the use of antihypertensive treatments is an important confounder; two prospective studies ${ }^{22,30}$ and five cross-sectional studies ${ }^{30,36,44,47,49}$ did not have this variable; in our stratified analysis, the effect was attenuated when studies took account of antihypertensive medication, but 
the negative association between decreased 25OHD and hypertension remained significant, and in addition, studies which took account of blood pressure medications did not have heterogeneity.

\section{Measurement of 25OHD levels}

There has been debate over the measurement of 25OHD in blood samples and its variability. ${ }^{58}$ HPLC has been considered the "gold standard" but as can be seen in the present stratifications, RIA was by far the most commonly used method. There were differences between these methods, which could well explain some of the heterogeneity detected in our meta-analyses. A recent paper has reported good similarity between these various methods, ${ }^{71}$ and with the exception of HPLC, all assay methods produced significant associations.

A

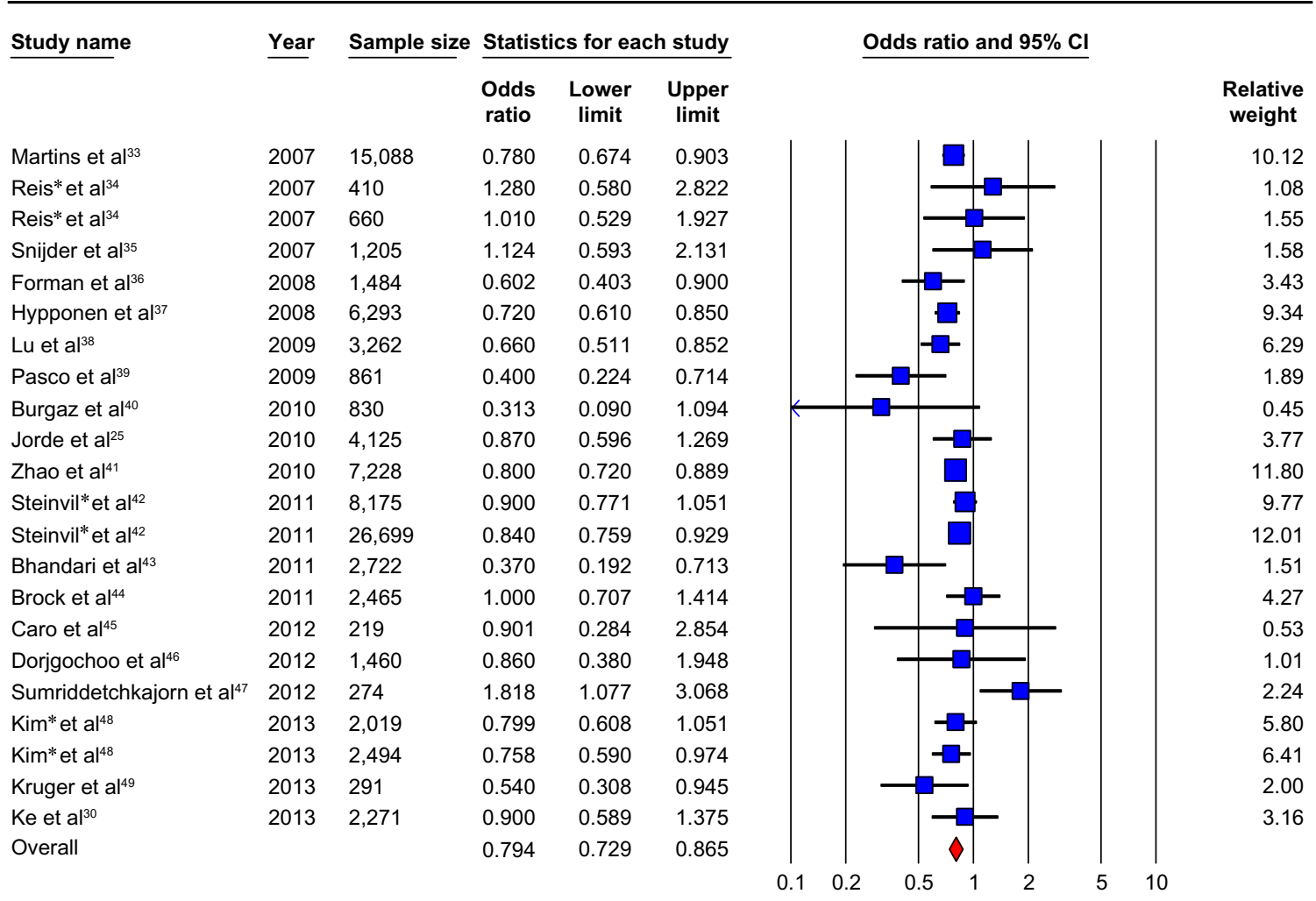

B

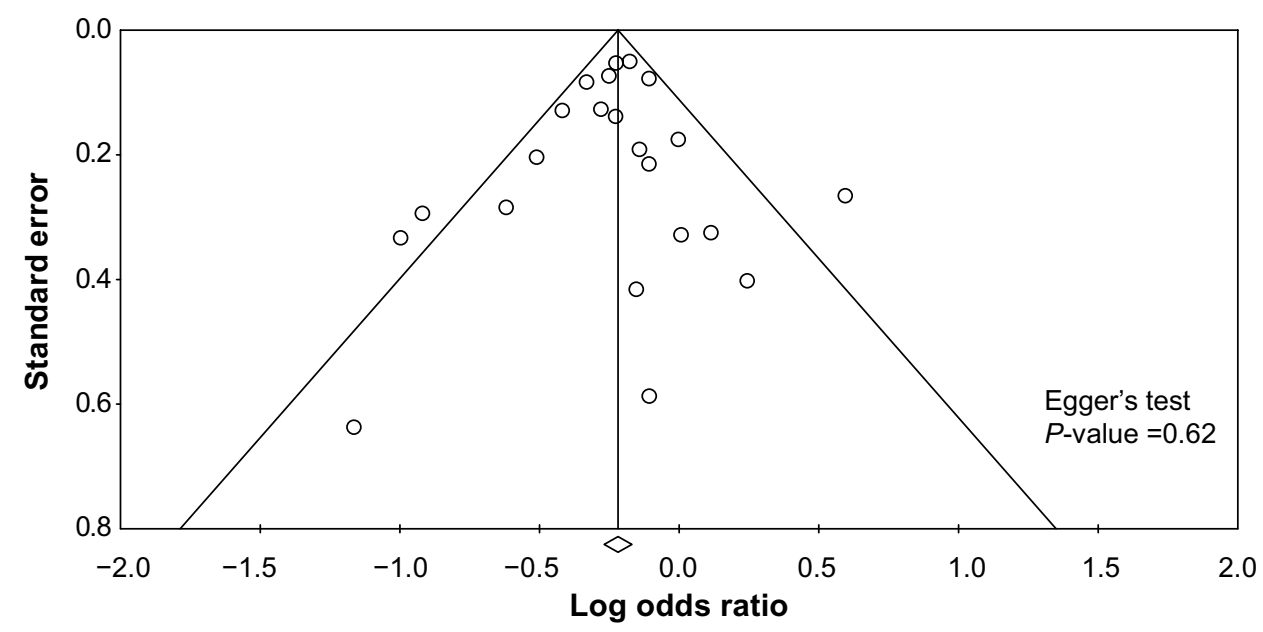

Figure 3 (Continued) 
C

\begin{tabular}{|c|c|c|c|c|c|c|c|c|}
\hline \multirow[t]{2}{*}{ Quality score } & \multirow[t]{2}{*}{ Study name } & \multirow[t]{2}{*}{ Year } & \multirow[t]{2}{*}{ Sample size } & \multicolumn{3}{|c|}{ Statistics for each study } & \multicolumn{2}{|l|}{ Odds ratio and $95 \% \mathrm{Cl}$} \\
\hline & & & & $\begin{array}{l}\text { Odds } \\
\text { ratio }\end{array}$ & $\begin{array}{l}\text { Lower } \\
\text { limit }\end{array}$ & $\begin{array}{l}\text { Upper } \\
\text { limit }\end{array}$ & & $\begin{array}{c}\text { Relative } \\
\text { weight }\end{array}$ \\
\hline 4.00 & Forman et $\mathrm{al}^{36}$ & 2008 & 1,484 & 0.602 & 0.403 & 0.900 & & 9.68 \\
\hline 4.00 & Steinvil ${ }^{*}$ et $a^{42}$ & 2011 & 8,175 & 0.900 & 0.771 & 1.051 & & 24.15 \\
\hline 4.00 & Steinvil* et al ${ }^{42}$ & 2011 & 26,699 & 0.840 & 0.759 & 0.929 & & 28.42 \\
\hline 4.00 & Brock et $\mathrm{al}^{44}$ & 2011 & 2,465 & 1.000 & 0.707 & 1.414 & & 11.82 \\
\hline 4.00 & Caro et $\mathrm{al}^{45}$ & 2012 & 219 & 0.901 & 0.284 & 2.854 & & 1.59 \\
\hline 4.00 & Dorjgochoo et $\mathrm{al}^{46}$ & 2012 & 1,460 & 0.860 & 0.380 & 1.948 & & 3.02 \\
\hline 4.00 & Sumriddetchkajorn et $a^{47}$ & 2012 & 274 & 1.818 & 1.077 & 3.068 & & 6.51 \\
\hline 4.00 & Kruger et $\mathrm{al}^{49}$ & 2013 & 291 & 0.540 & 0.308 & 0.945 & & 5.83 \\
\hline 4.00 & Ke et $\mathrm{al}^{30}$ & 2013 & 2,271 & 0.900 & 0.589 & 1.375 & & 8.99 \\
\hline 4.00 & & & & 0.872 & 0.751 & 1.012 & & \\
\hline 5.00 & Reis* et al ${ }^{34}$ & 2007 & 410 & 1.280 & 0.580 & 2.822 & & 3.27 \\
\hline 5.00 & Reis* et al ${ }^{34}$ & 2007 & 660 & 1.010 & 0.529 & 1.927 & & 4.65 \\
\hline 5.00 & Hypponen et $\mathrm{al}^{37}$ & 2008 & 6,293 & 0.720 & 0.610 & 0.850 & & 22.16 \\
\hline 5.00 & Lu et $a^{38}$ & 2009 & 3,262 & 0.660 & 0.511 & 0.852 & & 16.24 \\
\hline 5.00 & Pasco et $\mathrm{al}^{39}$ & 2009 & 861 & 0.400 & 0.224 & 0.714 & & 5.58 \\
\hline 5.00 & Burgaz et $\mathrm{al}^{40}$ & 2010 & 830 & 0.313 & 0.090 & 1.094 & & 1.40 \\
\hline 5.00 & Jorde et $\mathrm{al}^{25}$ & 2010 & 4,125 & 0.870 & 0.596 & 1.269 & & 10.50 \\
\hline 5.00 & Bhandari et al ${ }^{43}$ & 2011 & 2,722 & 0.370 & 0.192 & 0.713 & & 4.53 \\
\hline 5.00 & Kim* et al $\left.\right|^{48}$ & 2013 & 2,019 & 0.799 & 0.608 & 1.051 & & 15.18 \\
\hline 5.00 & Kim* et $\mathrm{al}^{48}$ & 2013 & 2,494 & 0.758 & 0.590 & 0.974 & & 16.49 \\
\hline 5.00 & & & & 0.713 & 0.613 & 0.829 & & \\
\hline 6.00 & Martins et $\mathrm{al}^{33}$ & 2007 & 15,088 & 0.780 & 0.674 & 0.903 & & 33.76 \\
\hline 6.00 & Snijder et $\mathrm{al}^{35}$ & 2007 & 1,205 & 1.124 & 0.593 & 2.131 & & 1.77 \\
\hline 6.00 & Zhao et al ${ }^{41}$ & 2010 & 7,228 & 0.800 & 0.720 & 0.889 & & 64.47 \\
\hline 6.00 & & & & 0.798 & 0.733 & 0.869 & X & \\
\hline Overall & & & & 0.795 & 0.744 & 0.849 & & \\
\hline & & & & & & & 0.5 & \\
\hline
\end{tabular}

D

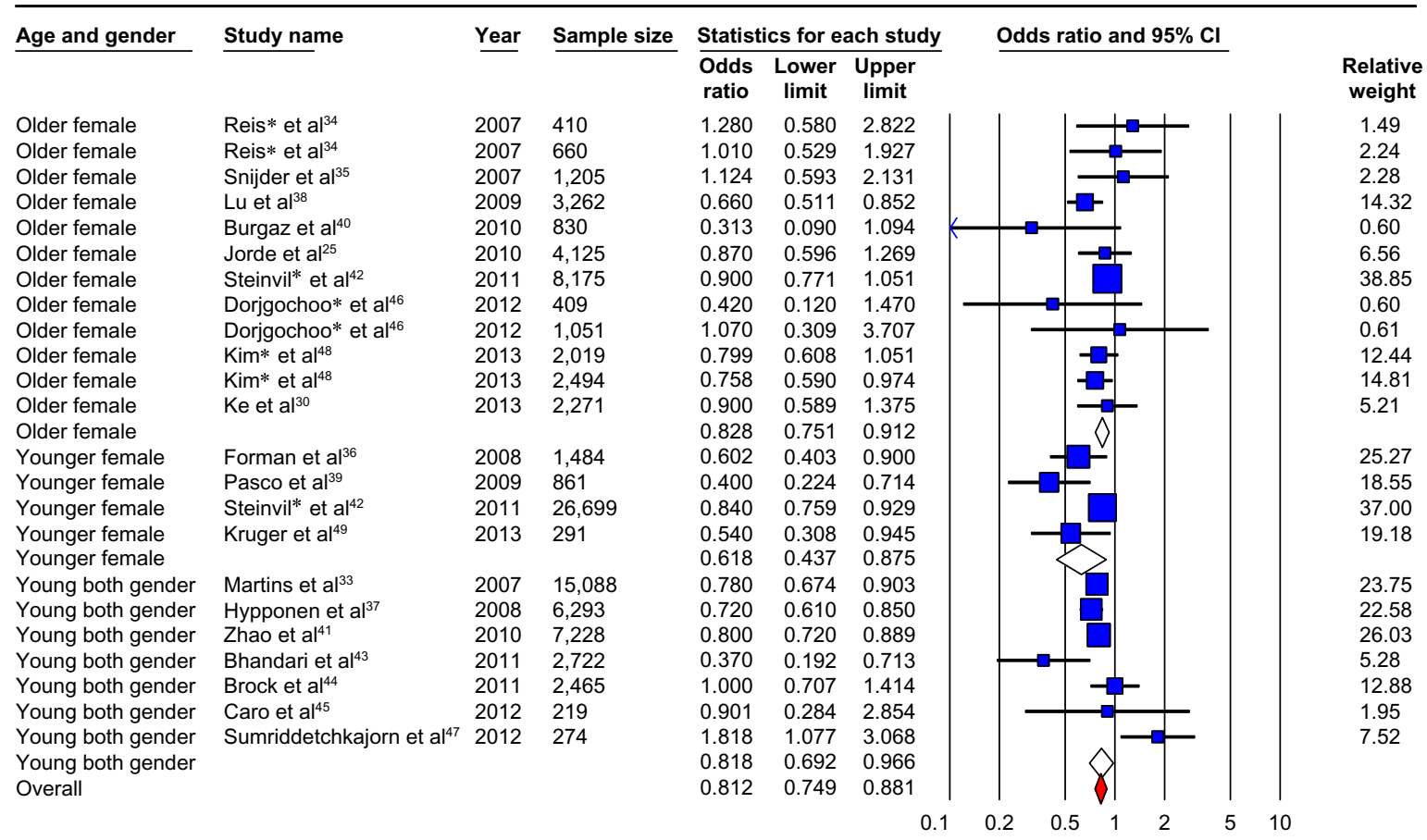

Figure 3 Cross-sectional studies.

Notes: (A) Cross-sectional studies of vitamin D and hypertension risk: the effect of higher vitamin D (measured as quartiles of $25 \mathrm{OHD}$ ) on hypertension risk expressed as odds ratio (OR) for individual studies (blue) and the calculated overall OR (red). (B) Funnel plot showing standard error by log OR for the cross-sectional studies. (C) Crosssectional studies of vitamin $D$ and hypertension risk sub-groups: the effect of higher vitamin $D$ (measured as quartiles of $25 \mathrm{OHD}$ ) on hypertension risk stratified by quality score expressed as OR for individual studies (blue), sub-group total(white) and the calculated overall OR (red). (D) Cross-sectional studies of vitamin D and hypertension risk sub-groups: the effect of higher vitamin D (measured as quartiles of $25 \mathrm{OHD}$ ) on hypertension risk stratified by age and gender expressed as OR for individual studies (blue), sub-groups total (white) and the calculated overall OR (red). *Studies published in one paper.

Abbreviation: $\mathrm{Cl}$, confidence Interval. 


\section{Season of blood draw and measurement of $25 \mathrm{OHD}$}

It is well established that $25 \mathrm{OHD}$ concentrations and hypertension are associated with season. ${ }^{72,73}$ When studies that adjusted for season were compared with studies that did not take seasonality into account (usually by date of draw of the blood samples), the association between low 25OHD and hypertension risk remained significant. Only one prospective study ${ }^{22}$ did not adjust for season, and in cross-sectional studies, most studies appear to have taken season into account ${ }^{30,33-40,44,46}$ but not others. In our stratification analysis, taking season into account did reduce the effect size but not significance.

\section{Deficiency vs sufficiency of vitamin $D$ status}

There has been some discussion that the effect of 25OHD on hypertension risk may be more potent in those who are originally vitamin D deficient vs sufficient. ${ }^{7,8,27}$ The opposite effect was seen in these data in that a significant effect was only seen in those who had sufficiency, not deficiency, although it should be pointed out that the deficiency studies were fewer in number and in size. In addition, when those who were older and had deficiency were investigated in prospective studies, the effect became nonsignificant and attenuated in cross-sectional studies ( $87 \%$ of total data).

\section{Confounding by healthy lifestyle variables}

It has been suggested that confounding by health lifestyle variables may account for the associations seen between higher 25OHD and less hypertension. ${ }^{64}$ In particular, BMI, as a marker of obesity, is an independent risk factor for both vitamin D deficiency ${ }^{74-77}$ and hypertension. ${ }^{78-83} \mathrm{In}$ fact, earlier investigators did not adjust for BMI in their hypertension and 25OHD analyses as they considered it to be a mediating factor and part of the causal chain. ${ }^{50}$ More recently, Mendelian analysis has confirmed that obesity is a "cause" of vitamin D deficiency, not the other way around, ${ }^{84}$ and recent studies have inferred that the effect of BMI may be due to a body dilution effect. ${ }^{85} \mathrm{In}$ this analysis, only $15 \%$ of studies (five) did not adjust for BMI, and adjustment did make the associations smaller in effect; they nevertheless remained significant. Thus, two recent Mendelian randomization studies investigating the relationship between adiposity and vitamin $\mathrm{D}$ concentrations have confirmed this important mediating effect of obesity and vitamin $\mathrm{D}^{84,86}$

\section{Potential mechanisms of action to explain a protective effect of vitamin $D$ in hypertension}

As we have summarized above, there are a number of confounding mechanisms, which could explain an association between low $25 \mathrm{OHD}$ concentrations and high blood pressure. In particular, being overweight or obese is known to be linked to low vitamin D concentrations and to higher readings for blood pressure using a normal sphygmomanometer cuff. 87,88

There are also a number of plausible physiological mechanisms to explain why low vitamin D status might lead to elevated blood pressure. The active hormone of vitamin $\mathrm{D}, 1,25 \mathrm{D}$, has been shown to decrease the expression of the renin gene through a vitamin $\mathrm{D}$ receptor-dependent mechanism, thus decreasing both renin and angiotensin II concentrations. ${ }^{89,90}$ This mechanism results in hypertension in mice, in which the capacity to produce $1,25 \mathrm{D}$ is knocked out, ${ }^{90}$ or in mice that lack the vitamin D receptor. ${ }^{89}$ A negative association between 25OHD, 1,25D concentrations, and plasma renin activity in patients with hypertension has been reported ${ }^{91}$ and in patients with chronic heart failure, a short course of vitamin D treatment resulted in a significant decrease in plasma renin activity and concentration. ${ }^{92}$ Vitamin D deficiency also results in higher than normal concentrations of PTH. High levels of PTH have long been associated with elevated blood pressure, reversed by parathyroid gland removal in mice, ${ }^{93}$ while treatment of older female normotensive women with vitamin $\mathrm{D}$ and calcium reduced blood pressure and parathyroid hormone, more effectively than calcium alone. ${ }^{94}$ There is also considerable evidence from both animal and human studies that the vitamin D system affects vascular endothelial function in several ways, including affecting vascular stiffness, oxidative stress, and through upregulation of endothelial nitric oxide syntheses. ${ }^{95-99}$

\section{Limitations}

Our study also has several strengths and limitations as do all meta-analyses of observational studies.

Inadequate control for confounders may bias the results in either direction, toward exaggeration or underestimation of the estimates, as such residual or unknown confounding cannot be excluded as a potential explanation for the observed findings. In this analysis, stratification has attempted to answer some of these concerns (Tables 2 and 4).

As was the case with Kunutsor et al, ${ }^{8}$ it was not possible to correct the estimates for within-individual variation in 
Table 5 Characteristics of studies reporting linear relationship between blood pressure and 25OHD

\begin{tabular}{|c|c|c|c|}
\hline $\begin{array}{l}\text { Author (year), }{ }^{\text {reference }} \text { study and year of } \\
\text { conduct, quality score }{ }^{\dagger}\end{array}$ & Country, study sample & $\begin{array}{l}\text { Gender, age (range or } \\
\text { mean } \pm \text { SD) (years) }\end{array}$ & $\begin{array}{l}\text { Sample } \\
\text { size, assay }\end{array}$ \\
\hline Scragg et al (2007),,$^{50}$ NHANES III: 1988-1994, 6 & US, general population & $48 \%$ male, $20-80+$ & I2,644, RIA \\
\hline Hintzpeter et al (2008), ${ }^{51}$ GNHIES 1998, 6 & Germany, general population & $44 \%$ male, $18-79$ & 4,030, CLIA \\
\hline Almirall et al $(2010),{ }^{52}$ D'AVIS study 2006,5 & Spain, general population & $47 \%$ male, $64-93$ & 237, RIA \\
\hline $\begin{array}{l}\text { Fraser et al }(2010),{ }^{53} \text { NHANES } 200 I-2002, \\
2003-2004,2005-2006,7\end{array}$ & US, general population & $48 \%$ male, $20-80+$ & $3,958, \mathrm{RIA}$ \\
\hline Griffin (201 I), ${ }^{23}$ MBHMS 1992-1993, 6 & US, general population & I00\% female, $22-44$ & $4 \mid 3, \mathrm{RIA}$ \\
\hline Kim et al $(2010),{ }^{26}$ Chungju city $2003-2007,5$ & Korea, general population & $38 \%$ male, $66 \pm 9$ & I,330, CLIA \\
\hline Li et al $(2012),{ }^{54}$ Dali city 2010,5 & $\begin{array}{l}\text { People's Republic of China, factory } \\
\text { employees }\end{array}$ & $60 \%$ male, $20-83$ & I,206, RIA \\
\hline Jungert et al (2012), ${ }^{55}$ GISELA 2008, 5 & Germany, general population & $32 \%$ male, $66-96$ & I32, ECLIA \\
\hline Skaaby et al (2012), ${ }^{32}$ Inter99 1999-2004, 6 & Denmark, general population & $50 \%$ male, $30-60$ & 4,330, HPLC \\
\hline Margolis et al (2012), ${ }^{27} \mathrm{WHI}$ 1993-1998, 5 & US, general population & $100 \%$ male, $50-79$ & 2,153, CLIA \\
\hline Kim et al $(2013),{ }^{48} \mathrm{KNHANES} 2009-2010,5$ & Korea, general population & $45 \%$ male, $50-90$ & $4,5 \mid 3, \mathrm{RIA}$ \\
\hline Sakamoto et al (2013), ${ }^{56}$ AHS2 2003-2007, 5 & US, general population & $48 \%$ male, $30-95$ & 568, RIA \\
\hline Wang et al (2013), ${ }^{29}$ PHS I982-2009, 5 & US, physicians & $100 \%$ male, $40-84$ & 660, RIA \\
\hline Sulistyoningrum et al (2013), ${ }^{57}$ M-CHAT 2006, 5 & Canada, general population & $48 \%$ male, $30-65$ & 687, RIA \\
\hline
\end{tabular}

Note: ${ }^{\dagger} Q u a l i t y$ score based on Newcastle-Ottawa Scale. $* P<0.05$

Abbreviations: NHANES, National Health and Nutrition Examination Survey; GNHIES, German National Health Interview and Examination Survey; D'AVIS, designed to analyze the prevalence of hypovitaminosis D; MBHMS, Michigan Bone Health and Metabolism; GISELA, Giessener Senioren Langzeitstudie; WHI, Women's Health Initiative; KNHANES, Korea National Health and Nutrition Examination Survey; AHS2, Adventist Health Study-2; PHS, Physicians' Health Study; M-CHAT, Multi-cultural Community Health Assessment Trial; 25OHD, 25-hydroxyvitamin D; SD, standard deviation; US, United States; BP, blood pressure; RIA, radioimmunoassay; SBP, systolic blood pressure; DBP, diastolic blood pressure; PA, physical activity; CLIA, chemiluminescence immunoassay; HTN, hypertension; BMI, body mass index; ECLIA, electro-chemiluminescence immunoassay; TBF, total body fat; WHR, waist-hip ratio; HPLC, high-pressure liquid chromatography; WC, waist circumference; NA, not available.

vitamin D levels over time or across seasons, because data involving repeat measurements were not reported. Although there are reports that $25 \mathrm{OHD}$ exhibits low within-individual variability $\left(r^{2}=0.87\right.$ between measured 25OHD levels after several years), ${ }^{100,101}$ studies are still needed with serial measurements of vitamin $\mathrm{D}$.

Another limitation is that 19 of the studies included had a cross-sectional design, which cannot exclude the possibility of reverse causality.

Despite the general nature of these cohorts, it must be noted that our and any other study investigating 25OHD levels are only reporting on those who gave blood, not the entire cohort (average response rate $=65 \%$ ).
Though the meta-analysis was very comprehensive, it was, as are all meta-analyses based on data from published reports, preventing the undertaking of adjustment for confounding and assessment of potential interactions. As has recently been discussed, meta-analyses are only as accurate as the veracity of included data. ${ }^{102}$ Thus, collaborative pooling of individual participant data from cohort studies should be conducted for more detailed analyses under a broader range of circumstances as has been done in recent Mendelian analyses. ${ }^{84,103}$

\section{Strengths}

In this analysis, ten prospective studies, which are better at being able to determine the role of blood 25OHD concentrations in 


\begin{tabular}{|c|c|c|c|c|}
\hline \multirow{2}{*}{$\begin{array}{l}\text { Mean or \% 25OHD } \\
(\mathrm{nmol} / \mathrm{L})\end{array}$} & \multirow{2}{*}{$\begin{array}{l}25 \mathrm{OHD}(\mathrm{nmol} / \mathrm{L}) \\
\text { comparison }\end{array}$} & \multicolumn{2}{|c|}{ Effects of $250 \mathrm{OHD}$} & \multirow[t]{2}{*}{ Confounders } \\
\hline & & Primary endpoint: BP & Linear or logistic regression & \\
\hline $\begin{array}{l}\text { Male: } 78 ; \\
\text { female: } 73\end{array}$ & Continuous & $\downarrow \mathrm{SBP}, \downarrow \mathrm{DBP}$ & SBP: $\beta=-0.023^{*}, \mathrm{DBP}: \beta=-0.027^{*}$ & $\begin{array}{l}\text { Age, gender, PA, ethnicity, } \\
\text { season }\end{array}$ \\
\hline $45,57 \%<50$ & $\begin{array}{l}\text { Per } 10 \mathrm{nmol} / \mathrm{L} \uparrow \\
\text { vitamin } \mathrm{D}\end{array}$ & $\downarrow \mathrm{BP}$ & $\begin{array}{l}\text { Male: } 0.97(0.94-0.99) \\
\text { Female: } 0.96(0.93-0.99)\end{array}$ & Age, season, HTN treatment \\
\hline $86 \%<75$ & Continuous & $\downarrow \mathrm{SBP}, \downarrow \mathrm{DBP}$ & SBP: $\beta=-0.29^{*}, \mathrm{DBP}: \beta=-0.15^{*}$ & $\begin{array}{l}\text { Age, gender, BMI, HTN } \\
\text { treatment, diabetes }\end{array}$ \\
\hline 57 & Continuous & $\downarrow \mathrm{SBP}, \leftrightarrow \mathrm{DBP}$ & SBP: $\beta=-0.032^{*}, \mathrm{DBP}: \beta=-0.002$ & $\begin{array}{l}\text { Age, gender, BMI, ethnicity, } \\
\text { season, HTN treatment, } \\
\text { diabetes status }\end{array}$ \\
\hline $59,81 \%<75$ & Continuous & $\leftrightarrow \mathrm{SBP}, \leftrightarrow \mathrm{DBP}$ & SBP: $\beta=-0.650, \mathrm{DBP}: \mathrm{P}>0.05$ & $\begin{array}{l}\text { Age, \% body fat, season, HTN } \\
\text { treatment }\end{array}$ \\
\hline 43 & Continuous & $\downarrow \mathrm{SBP}, \downarrow \mathrm{DBP}$ & SBP: $\beta=-0.150^{*}, \mathrm{DBP}: \beta=-0.120^{*}$ & Age, gender, BMI, season \\
\hline $75 \%<70$ & $\begin{array}{l}\text { Per } 10 \mathrm{nmol} / \mathrm{L} \uparrow \\
\text { vitamin } \mathrm{D}\end{array}$ & $\leftrightarrow \mathrm{BP}$ & $1.00(0.98-1.02)$ & $\begin{array}{l}\text { Age, gender, BMI, HTN } \\
\text { treatment }\end{array}$ \\
\hline $\begin{array}{l}21 \% \text { male }<50 \\
23 \% \text { female }<50\end{array}$ & Continuous & $\begin{array}{l}\uparrow \mathrm{SBP}(\text { male), } \leftrightarrow \text { DBP } \\
\text { (male), } \leftrightarrow \text { SBP (female), } \\
\leftrightarrow \text { DBP (female) }\end{array}$ & $\begin{array}{l}\text { SBP: } \beta=-0.356^{*} \text { male, DBP: } \\
\beta=-0.198 \text { male, SBP: } \beta=-0.046 \\
\text { female, DBP: } \beta=-0.104 \text { female }\end{array}$ & $\begin{array}{l}\text { Age, TBF, WHR, HTN } \\
\text { treatment }\end{array}$ \\
\hline 47, female $=50$ & $\begin{array}{l}\text { Per } 10 \mathrm{nmol} / \mathrm{L} \uparrow \\
\text { vitamin } D\end{array}$ & $\leftrightarrow \mathrm{BP}$ & $\mathrm{I} .0 \mathrm{I}(0.97, \mathrm{I} .05)$ & Age, gender, BMI, PA, season \\
\hline $54 \%<50$ & Continuous & $\uparrow \mathrm{BP}$ & $\begin{array}{l}\text { HTN\% vs } 25 \mathrm{OHD} \text { quartile, } \\
\text { Chi-square } P<0.0 \text { I }\end{array}$ & NA \\
\hline $49,53 \%<50$ & Continuous & $\downarrow \mathrm{SBP}, \downarrow \mathrm{DBP}$ & $\begin{array}{l}\text { SBP: } \beta=-0.128 \text { male, DBP: } \\
\beta=-0.087^{*} \text { male, } \\
\text { SBP: } \beta=-0.156^{*} \text { female, } \\
\text { DBP: } \beta=-0.099^{*} \text { female }\end{array}$ & $\begin{array}{l}\text { Age, weight, WC, PA, HTN } \\
\text { treatment, diabetes status }\end{array}$ \\
\hline $\begin{array}{l}\text { Whites } 15 \%<50 \\
\text { Black } 55 \%<50\end{array}$ & Continuous & $\uparrow \mathrm{SBP}, \leftrightarrow \mathrm{DBP}$ & $\begin{array}{l}\text { SBP: } \beta=-0.250^{*} \text { (Whites), } \\
\beta=0.080 \text { (Black), DBP: } \\
\beta=-0.1 \text { I } 0 \text { (total) }\end{array}$ & $\begin{array}{l}\text { Age, gender, BMI, HTN } \\
\text { treatment }\end{array}$ \\
\hline $\begin{array}{l}\text { Summer: } 77, \\
\text { Winter: } 56\end{array}$ & Continuous & $\leftrightarrow \mathrm{SBP}, \leftrightarrow \mathrm{DBP}$ & $\begin{array}{l}\text { No significant mean } \\
250 H D \text { difference }\end{array}$ & $\begin{array}{l}\text { Age, BMI, PA, ethnicity, season, } \\
\text { diabetes status }\end{array}$ \\
\hline $39,71 \%<50$ & Continuous & $\downarrow \mathrm{SBP}, \downarrow \mathrm{DBP}$ & $\begin{array}{l}\text { SBP: } \beta=-0.098^{*} \\
\text { DBP: } \beta=-0.104^{*}\end{array}$ & Age, gender, BMI, PA, ethnicity \\
\hline
\end{tabular}

the cause of hypertension, were included. Most of the studies in this meta-analysis were designed to specifically investigate the effect of blood 25OHD concentrations on the risk of hypertension in a general population. Twenty-two studies had as the main focus hypertension and the other seven had metabolic syndrome or heart diseases. More cohort studies specifically designed to investigate this question are needed with long follow-up time as the study by Jorde et $\mathrm{al}^{25}$ has presented.

This is the largest meta-analysis of both prospective and cross-sectional associations conducted to date (excluding data from dietary studies and nested casecontrol studies) and provides precise estimates of the magnitude of the association of hypertension risk with vitamin D levels.
This present meta-analysis included studies that had recruited participants from mainly general populations, therefore reducing any effects of clinically evident preexisting disease on vitamin D levels.

In this meta-analysis, the eligible studies were carried out in many study sites and countries, which enhances generalization of our findings. Despite the general nature of these cohorts, it must be noted that our and any other study investigating $25 \mathrm{OHD}$ levels are only reporting on those who gave blood, not the entire cohort (average response rate $=65 \%$, ranging from $10 \%$ to $100 \%$ ).

As with any meta-analysis of published studies, ${ }^{7,8}$ publication bias is of concern as small studies with null results tend not to be published; however, in both prospective and 
cross-sectional studies, there was no evidence of publication bias.

Sensitivity and stratification analysis conducted with these data attempted to examine the impact of known hypertension risk factors and $25 \mathrm{OHD}$ confounders.

Findings from our review suggest the possibility of a causal relationship, but establishing this requires robust evidence from other study designs such as clinical trials and Mendelian analyses. Mendelian analysis may shed light on whether vitamin D could be directly causal in hypertension risk. A large percentage of the variability in $25 \mathrm{OHD}$ levels is explained by genetic factors. Heritability of $25 \mathrm{OHD}$ levels has been estimated to be as high as $80 \%$. The reason for the advantages of Mendelian randomization is that individual genotypes are assigned randomly at meiosis; thus, the effect of genetics on diseases is generally unaffected by confounding or reverse causality. Two genotypes CYP2R1 and CHCR7 have been found to function upstream of $25 \mathrm{OHD}$ production and affect the vitamin $\mathrm{D}$ metabolic system and subsequent substrate availability. ${ }^{103}$ These genotypes affect vitamin D metabolism as proxy markers for lifelong differences in vitamin D status. Thus Vimaleswaran et $\mathrm{a}^{103}$ in a Mendelian analysis of 108,173 individuals from 35 studies used these genetic variables to test for a causal association with blood pressure and hypertension, which showed that each $10 \%$ increase in genetically instrumented plasma $25 \mathrm{OHD}$ concentration was associated with a significant $8 \%$ reduced odds of hypertension $(\mathrm{OR}=0.92(0.87-0.97))$ as well as reduced $\operatorname{SBP}(P=0.003)$ and DBP $(P=0.01)$.

However, there is still a need to see what dosages of $25 \mathrm{OHD}$ affect hypertension risk via RCTs, and as mentioned previously, the most recent meta-analysis of vitamin D supplementation and hypertension has observed no evidence to support an effect of vitamin D supplementation on hypertension risk. Many of the RCTs are small studies on specialized subjects (often from clinics), and there are large differences in duration and doses of 25OHD. ${ }^{14}$ As many commentators have urged, ${ }^{9-12,62,63,104}$ there is a need for carefully designed large RCTs with long-term treatment with optimum vitamin D dosages on blood pressure. The recent and continuing Vitamin D and OmegA-3 TriaL (VITAL), an RCT of 20,000 US men and women $>50$ years, is investigating whether taking daily supplements of vitamin D3 reduces the risk of CVDs in people without prior history of these diseases, which may help disentangle and clarify this situation. ${ }^{105}$

\section{Conclusion}

Despite the evidence of a consistent link between vitamin D and blood pressure, questions still remain in relation to the causality of this relationship. Findings from this systematic literature review suggest the possibility of a causal relationship, but several RCTs have attempted to address these questions but with inconsistent results. Further studies either combining and re-analyzing existing data from available cohort studies or conducting further Mendelian analysis are needed to determine whether this represents a causal association. In addition, large RCTs are needed to determine whether vitamin supplementation or therapy may be beneficial in the prevention or the treatment of hypertension.

\section{Disclosure}

RSM receives speaker fees from Amgen Pty Ltd and AbbVie Australia. The other authors report no conflict of interest in this work.

\section{References}

1. Guo F, He D, Zhang W, Walton RG. Trends in prevalence, awareness, management, and control of hypertension among United States adults, 1999 to 2010. J Am Coll Cardiol. 2012;60(70):599-606.

2. World Health Organization. Global Status Report on Non-Communicable Diseases 2010. Geneva: World Health Organization; 2011.

3. Ibrahim MM, Damasceno A. Hypertension in developing countries. Lancet. 2012;380(9841):611-619.

4. Cutler JA, Sorlie PD, Wolz M, Thom T, Fields LE, Roccella EJ. Trends in hypertension prevalence, awareness, treatment, and control rates in United States adults between 1988-1994 and 1999-2004. Hypertension. 2008;52(5):818-827.

5. Lavie CJ, Dinicolantonio JJ, Milani RV, O'Keefe JH. Vitamin D and cardiovascular health. Circulation. 2013;128(22):2404-2406.

6. Witham MD, Nadir MA, Struthers AD. Effect of vitamin D on blood pressure: a systematic review and meta-analysis. $J$ Hypertens. 2009;27(10):1948-1954.

7. Burgaz A, Orsini N, Larsson SC, Wolk A. Blood 25hydroxyvitamin $\mathrm{D}$ concentration and hypertension: a meta-analysis. J Hypertens. 2011;29(4):636-645.

8. Kunutsor SK, Apekey TA, Steur M. Vitamin D and risk of future hypertension: meta-analysis of 283,537 participants. Eur J Epidemiol. 2013;28(3):205-221.

9. Lips P. Vitamin D physiology. Prog Biophys Mol Biol. 2006;92(1): 4-8.

10. Ross AC, Manson JE, Abrams SA, et al. The 2011 report on dietary reference intakes for calcium and vitamin D from the Institute of medicine: what clinicians need to know. $J$ Clin Endocrinol Metab. 2011;96(1):53-58.

11. Rosen CJ, Adams JS, Bikle DD, et al. The nonskeletal effects of vitamin D: an endocrine society scientific statement. Endocr Rev. 2012;33(3):456-492.

12. Weng S, Sprague JE, Oh J, et al. Vitamin D deficiency induces high blood pressure and accelerates atherosclerosis in mice. PLoS One. 2013;8(1):e54625.

13. Cozzolino M, Ketteler M, Zehnder D. The vitamin D system: a crosstalk between the heart and kidney. Eur J Heart Fail. 2010;12(10): 1031-1041.

14. Kunutsor SK, Burgess S, Munroe PB, Khan H. Vitamin D and high blood pressure: causal association or epiphenomenon? Eur J Epidemiol. 2014;29(1):1-14.

15. Moher D, Liberati A, Tetzlaff J, Altman DG. Preferred reporting items for systematic reviews and meta-analyses: the PRISMA statement. Ann Intern Med. 2009;151(4):264-269, W64. 
16. Rueda S, Fernandez-Fernandez C, Romero F, Martinez de Osaba J, Vidal J. Vitamin D, PTH, and the metabolic syndrome in severely obese subjects. Obes Surg. 2008;18(2):151-154.

17. Wu PW, Rhew EY, Dyer AR, et al. 25-hydroxyvitamin D and cardiovascular risk factors in women with systemic lupus erythematosus. Arthritis Rheum. 2009;61(10):1387-1395.

18. Ford ES, Ajani UA, McGuire LC, Liu S. Concentrations of serum vitamin D and the metabolic syndrome among US adults. Diabetes Care. 2005;28(5):1228-1230.

19. Wells GA, Shea B, O'Connell D, et al. The Newcastle-Ottawa Scale (NOS) for Assessing the Quality of Nonrandomised Studies in Meta-Analyses. Ottawa, ON: Ottawa Hospital Research Institute; 2011.

20. Huedo-Medina TB, Sánchez-Meca J, Marin-Martinez F, Botella J. Assessing heterogeneity in meta-analysis. Psychol Methods. 2006;11(2):193.

21. Monroe J. Meta-analysis for observational studies: statistical methods for heterogeneity, publication bias and combining studies, Master of Science in Statistics, University of California Los Angeles, 2007.

22. Forman JP, Giovannucci E, Holmes MD, et al. Plasma 25-hydroxyvitamin D levels and risk of incident hypertension. Hypertension. 2007; 49(5):1063-1069.

23. Griffin FC, Gadegbeku CA, Sowers MR. Vitamin D and subsequent systolic hypertension among women. Am J Hypertens. 2011;24(3): 316-321.

24. Anderson JL, May HT, Horne BD, et al. Intermountain Heart Collaborative (IHC) Study Group. Relation of vitamin D deficiency to cardiovascular risk factors, disease status, and incident events in a general healthcare population. Am J Cardiol. 2010;106(7):963-968.

25. Jorde R, Figenschau Y, Emaus N, Hutchinson M, Grimnes G. Serum 25-hydroxyvitamin D levels are strongly related to systolic blood pressure but do not predict future hypertension. Hypertension. 2010;55(3): 792-798.

26. Kim MK, Il Kang M, Won Oh K, et al. The association of serum vitamin D level with presence of metabolic syndrome and hypertension in middle-aged Korean subjects. Clin Endocrinol (Oxf). 2010;73(3):330-338.

27. Margolis KL, Martin LW, Ray RM, et al. Women's Health Initiative Investigators. A prospective study of serum 25-hydroxyvitamin D levels, blood pressure, and incident hypertension in postmenopausal women. Am J Epidemiol. 2012;175(1):22-32.

28. Gagnon C, Lu ZX, Magliano DJ, et al. Low serum 25-hydroxyvitamin D is associated with increased risk of the development of the metabolic syndrome at five years: results from a national, population-based prospective study (the Australian diabetes, obesity and lifestyle study: AusDiab). J Clin Transl Endocrinol. 2012;97(6):1953-1961.

29. Wang L, Ma J, Manson JE, Buring JE, Gaziano JM, Sesso HD. A prospective study of plasma vitamin D metabolites, vitamin D receptor gene polymorphisms, and risk of hypertension in men. Eur J Nutr. 2013;52(7):1771-1779.

30. Ke L, Graubard BI, Albanes D, et al. Hypertension, pulse, and other cardiovascular risk factors and vitamin D status in Finnish men. Am J Hypertens. 2013;26(8):951-956.

31. van Ballegooijen AJ, Kestenbaum B, Sachs MC, et al. Association of 25-Hydroxyvitamin D and parathyroid hormone with incident hypertension: MESA (multi-ethnic study of atherosclerosis). J Am Coll Cardiol. 2014;63(12):1214-1222.

32. Skaaby T, Husemoen LL, Pisinger C, et al. Vitamin D status and changes in cardiovascular risk factors: a prospective study of a general population. Cardiology. 2012;123(1):62-70.

33. Martins D, Wolf M, Pan D, et al. Prevalence of cardiovascular risk factors and the serum levels of 25-hydroxyvitamin D in the United States: data from the third national health and nutrition examination survey. Arch Intern Med. 2007;167(11):1159-1165.

34. Reis JP, von Muhlen D, Kritz-Silverstein D, Wingard DL, Barrett-Connor E. Vitamin D, parathyroid hormone levels, and the prevalence of metabolic syndrome in community-dwelling older adults. Diabetes Care. 2007;30(6):1549-1555.
35. Snijder MB, Lips P, Seidell JC, et al. Vitamin D status and parathyroid hormone levels in relation to blood pressure: a populationbased study in older men and women. J Intern Med. 2007;261(6): $558-565$.

36. Forman JP, Curhan GC, Taylor EN. Plasma 25-hydroxyvitamin D levels and risk of incident hypertension among young women. Hypertension. 2008;52(5):828-832.

37. Hypponen E, Boucher BJ, Berry DJ, Power C. 25-hydroxyvitamin D, IGF-1, and metabolic syndrome at 45 years of age: a cross-sectional study in the 1958 British birth cohort. Diabetes. 2008;57(2): 298-305.

38. Lu L, Yu Z, Pan A, et al. Plasma 25-hydroxyvitamin D concentration and metabolic syndrome among middle-aged and elderly Chinese individuals. Diabetes Care. 2009;32(7):1278-1283.

39. Pasco JA, Henry MJ, Nicholson GC, Brennan SL, Kotowicz MA. Behavioural and physical characteristics associated with vitamin D status in women. Bone. 2009;44(6):1085-1091.

40. Burgaz A, Byberg L, Rautiainen S, et al. Confirmed hypertension and plasma 25(OH)D concentrations amongst elderly men. J Intern Med. 2010;269(2):211-218

41. Zhao G, Ford ES, Li C, Kris-Etherton PM, Etherton TD, Balluz LS Independent associations of serum concentrations of 25-hydroxyvitamin $\mathrm{D}$ and parathyroid hormone with blood pressure among US adults. J Hypertens. 2010;28(9):1821-1828.

42. Steinvil A, Leshem-Rubinow E, Berliner S, et al. Vitamin D deficiency prevalence and cardiovascular risk in Israel. Eur J Clin Invest. 2011;41(3):263-268.

43. Bhandari SK, Pashayan S, Liu IL, et al. 25-hydroxyvitamin D levels and hypertension rates. J Clin Hypertens (Greenwich). 2011;13(3): 170-177.

44. Brock KE, Huang WY, Fraser DR, et al. Diabetes prevalence is associated with serum 25-hydroxyvitamin D and 1,25-dihydroxyvitamin D in US middle-aged Caucasian men and women: a cross-sectional analysis within the prostate, lung, colorectal and ovarian cancer screening trial Br J Nutr. 2011;106(3):339-344.

45. Caro Y, Negrón V, Palacios C. Association between Vitamin D levels and blood pressure in a group of Puerto Ricans. $P R$ Health Sci J. 2012;31(3):123-129.

46. Dorjgochoo T, Ou Shu X, Xiang YB, et al. Circulating 25 hydroxyvitamin D levels in relation to blood pressure parameters and hypertension in the Shanghai Women's and Men's Health Studies. Br J Nutr. 2012;108(3):449-458.

47. Sumriddetchkajorn K, Chailurkit L, Thakkinstian A, Sritara P. Hypertension is statistically associated with higher body mass index but not with vitamin D level in a Thai population. Eur J Clin Nutr. 2012;66(3):405-407.

48. Kim H, Chung YE, Jung SC, et al. Independent associations of circulating 25-hydroxyvitamin D and parathyroid hormone concentrations with blood pressure among Koreans: the Korea national health and nutrition examination survey (KNHANES), 2009-2010. Calcif Tissue Int. 2013;93(6):549-555.

49. Kruger IM, Kruger MC, Doak CM, et al. The association of 25(OH)D with blood pressure, pulse pressure and carotid-radial pulse wave velocity in African women. PLoS One. 2013;8(1):e54554.

50. Scragg R, Sowers M, Bell C. Serum 25-hydroxyvitamin D, ethnicity, and blood pressure in the third national health and nutrition examination survey. Am J Hypertens. 2007;20(7):713-719.

51. Hintzpeter B, Mensink GB, Thierfelder W, Muller MJ, Scheidt-Nave C. Vitamin D status and health correlates among German adults. Eur J Clin Nutr. 2008;62(9):1079-1089.

52. Almirall J, Vaqueiro M, Bare ML, Anton E. Association of low serum 25-hydroxyvitamin D levels and high arterial blood pressure in the elderly. Nephrol Dial Transplant. 2010;25(2):503-509.

53. Fraser A, Williams D, Lawlor DA. Associations of serum 25-hydroxyvitamin D, parathyroid hormone and calcium with cardiovascular risk factors: analysis of 3 NHANES cycles (2001-2006). PLoS One. 2010;5(11):e13882. 
54. Li L, Yin X, Yao C, Zhu X, Wu X. Vitamin D, parathyroid hormone and their associations with hypertension in a Chinese population. PLoS One. 2012;7(8):e43344.

55. Jungert A, Roth HJ, Neuhauser-Berthold M. Serum 25-hydroxyvitamin D3, parathyroid hormone and blood pressure in an elderly cohort from Germany: a cross-sectional study. Nutr Metab (Lond). 2012;9(1):20.

56. Sakamoto R, Jaceldo-Sieg1 K, Haddad E, Oda K, Fraser GE, Tonstad S. Relationship of vitamin D levels to blood pressure in a biethnic population. Nutr Metab Cardiovasc Dis. 2013;23(8):776-784.

57. Sulistyoningrum DC, Gasevic D, Green TJ, Lear SA, Devlin AM. Adiposity and the relationship between vitamin $\mathrm{D}$ and blood pressure. Metabolism. 2013;62(12):1795-1802.

58. Ashwell M, Stone EM, Stolte H, et al. UK food standards agency workshop report: an investigation of the relative contributions of diet and sunlight to vitamin D status. Br J Nutr. 2010;104(4):603-611.

59. Kong J, Qiao G, Zhang Z, Liu SQ, Li YC. Targeted vitamin D receptor expression in juxtaglomerular cells suppresses renin expression independent of parathyroid hormone and calcium. Kidney Int. 2008;74(12): $1577-1581$.

60. Merke J, Hofmann W, Goldschmidt D, Ritz E. Demonstration of $1,25(\mathrm{OH}) 2$ vitamin D3 receptors and actions in vascular smooth muscle cells in vitro. Calcif Tissue Int. 1987;41(2):112-114.

61. Paxton GA, Teale GR, Nowson CA, et al. Australian and New Zealand Bone and Mineral Society; Osteoporosis Australia. Vitamin D and health in pregnancy, infants, children and adolescents in Australia and New Zealand: a position statement. Med J Aust. 2013;198(3): 142-143.

62. Autier P, Boniol M, Pizot C, Mullie P. Vitamin D status and ill health: a systematic review. Lancet Diabetes Endocrinol. 2014;2(1):76-89.

63. Schnatz PF, Manson JE. Vitamin D and cardiovascular disease: an appraisal of the evidence. Clin Chem. 2014;60(4):600-609.

64. Vitamin D: chasing a myth? Lancet Diabetes Endocrinol. 2014;2(1):1.

65. Brock KE, Ke L, Tseng M, et al. Vitamin D status is associated with sun exposure, vitamin D and calcium intake, acculturation and attitudes in immigrant East Asian women living in Sydney. J Steroid Biochem Mol Biol. 2013;136:214-217.

66. Ke L, Ho J, Feng J, et al. Modifiable risk factors including sunlight exposure and fish consumption are associated with risk of hypertension in a large representative population from Macau. J Steroid Biochem Mol Biol. 2014;144(pt A):152-155.

67. Ke L, Mason RS, Mpofu E, et al. Vitamin D and Parathyroid Hormone Status in a Representative Population Living in Macau, China. J Steroid Biochem Mol Biol. 2015. pii: S0960-0760(15)00031-X. doi: 10.1016/j. jsbmb.2015.01.019. [Epub ahead of print]

68. Lips P, van Schoor NM, de Jongh RT. Diet, sun and lifestyle as determinants of vitamin D status. Ann N Y Acad Sci. 2014;1317: 92-98.

69. Scragg R, Sowers M, Bell C. Serum 25-hydroxyvitamin D, diabetes, and ethnicity in the third national health and nutrition examination survey. Diabetes Care. 2004;27(12):2813-2818.

70. Jang H, Koo FK, Ke L, et al. Culture and sun exposure in immigrant East Asian women living in Australia. Women Health. 2013;53(5): 504-518.

71. Wallace AM, Gibson S, de la Hunty A, Lamberg-Allardt C, Ashwell M. Measurement of 25-hydroxyvitamin D in the clinical laboratory: current procedures, performance characteristics and limitations. Steroids. 2010;75(7):477-488.

72. Burgaz A, Akesson A, Michaelsson K, Wolk A. 25-hydroxyvitamin D accumulation during summer in elderly women at latitude 60 degrees $\mathrm{N}$. J Intern Med. 2009;266(5):476-483.

73. Kamezaki F, Sonoda S, Tomotsune Y, Yunaka H, Otsuji Y. Seasonal variation in metabolic syndrome prevalence. Hypertens Res. 2010;33(6):568-572.

74. Sulistyoningrum DC, Green TJ, Lear SA, Devlin AM. Ethnic-specific differences in vitamin D status is associated with adiposity. PLoS One. 2012;7(8):e43159
75. Young KA, Engelman CD, Langefeld CD, et al. Association of plasma vitamin D levels with adiposity in Hispanic and African Americans. J Clin Endocrinol Metab. 2009;94(9):3306-3313.

76. Cheng S, Massaro JM, Fox CS, et al. Adiposity, cardiometabolic risk, and vitamin D status: the Framingham Heart Study. Diabetes. 2010;59(1):242-248.

77. Caron-Jobin M, Morisset AS, Tremblay A, Huot C, Legare D, Tchernof A. Elevated serum 25(OH)D concentrations, vitamin D, and calcium intakes are associated with reduced adipocyte size in women. Obesity (Silver Spring). 2011;19(7):1335-1341.

78. Fox CS, Massaro JM, Hoffmann U, et al. Abdominal visceral and subcutaneous adipose tissue compartments: association with metabolic risk factors in the Framingham Heart Study. Circulation. 2007;116(1):39-48.

79. Oka R, Miura K, Sakurai M, et al. Impacts of visceral adipose tissue and subcutaneous adipose tissue on metabolic risk factors in middle-aged Japanese. Obesity (Silver Spring). 2010;18(1):153-160.

80. Liu J, Fox CS, Hickson DA, et al. Impact of abdominal visceral and subcutaneous adipose tissue on cardiometabolic risk factors: the Jackson Heart Study. J Clin Endocrinol Metab. 2010;95(12):5419-5426.

81. Sironi AM, Gastaldelli A, Mari A, et al. Visceral fat in hypertension: influence on insulin resistance and beta-cell function. Hypertension. 2004:44(2):127-133.

82. Koh H, Hayashi T, Sato KK, et al. Visceral adiposity, not abdominal subcutaneous fat area, is associated with high blood pressure in Japanese men: the Ohtori study. Hypertens Res. 2011;34(5):565-572.

83. Lear SA, Chockalingam A, Kohli S, Richardson CG, Humphries KH. Elevation in cardiovascular disease risk in South Asians is mediated by differences in visceral adipose tissue. Obesity (Silver Spring). 2012;20(6):1293-1300.

84. Vimaleswaran KS, Berry DJ, Lu C, et al. Genetic Investigation of Anthropometric Traits-GIANT Consortium. Causal relationship between obesity and vitamin D status: bi-directional mendelian randomization analysis of multiple cohorts. PLoS Med. 2013;10(2): e1001383.

85. Gallagher JC, Yalamanchili V, Smith LM. The effect of vitamin D supplementation on serum 25(OH)D in thin and obese women. J Steroid Biochem Mol Biol. 2013;136:195-200.

86. Afzal S, Brondum-Jacobsen P, Bojesen SE, Nordestgaard BG. Vitamin D concentration, obesity, and risk of diabetes: a mendelian randomisation study. Lancet Diabetes Endocrinol. 2014;2(4):298-306.

87. Wortsman J, Matsuoka LY, Chen TC, Lu Z, Holick MF. Decreased bioavailability of vitamin D in obesity. Am J Clin Nutr. 2000;72(3): 690-693.

88. Timpson NJ, Harbord R, Davey Smith G, Zacho J, Tybjaerg-Hansen A, Nordestgaard BG. Does greater adiposity increase blood pressure and hypertension risk?: mendelian randomization using the FTO/MC4R genotype. Hypertension. 2009;54(1):84-90.

89. LiYC, Kong J, Wei M, Chen ZF, Liu SQ, Cao LP. 1,25-Dihydroxyvitamin $\mathrm{D}(3)$ is a negative endocrine regulator of the renin-angiotensin system. J Clin Invest. 2002;110(2):229-238.

90. Zhou C, Lu F, Cao K, Xu D, Goltzman D, Miao D. Calcium-independent and 1,25(OH)2D3-dependent regulation of the renin-angiotensin system in 1alpha-hydroxylase knockout mice. Kidney Int. 2008;74(2): 170-179.

91. Resnick LM, Muller FB, Laragh JH. Calcium-regulating hormones in essential hypertension. Relation to plasma renin activity and sodium metabolism. Ann Intern Med. 1986;105(5):649-654.

92. Schroten NF, Ruifrok WP, Kleijn L, et al. Short-term vitamin D3 supplementation lowers plasma renin activity in patients with stable chronic heart failure: an open-label, blinded end point, randomized prospective trial (VitD-CHF trial). Am Heart J. 2013;166(2): 357-364e352.

93. Mann JF, Wiecek A, Bommer J, Ganten U, Ritz E. Effects of parathyroidectomy on blood pressure in spontaneously hypertensive rats. Nephron. 1987;45(1):46-52. 
94. Pfeifer M, Begerow B, Minne HW, Nachtigall D, Hansen C. Effects of a short-term vitamin $\mathrm{D}(3)$ and calcium supplementation on blood pressure and parathyroid hormone levels in elderly women. J Clin Endocrinol Metab. 2001;86(4):1633-1637.

95. Giallauria F, Milaneschi Y, Tanaka T, et al. Arterial stiffness and vitamin D levels: the Baltimore longitudinal study of aging. J Clin Endocrinol Metab. 2012;97(10):3717-3723.

96. Tare M, Emmett SJ, Coleman HA, et al. Vitamin D insufficiency is associated with impaired vascular endothelial and smooth muscle function and hypertension in young rats. J Physiol. 2011;589(pt 19): 4777-4786

97. Sachse A, Wolf G. Angiotensin II-induced reactive oxygen species and the kidney. J Am Soc Nephrol. 2007;18(9):2439-2446.

98. Andrukhova O, Slavic S, Zeitz U, et al. Vitamin D is a regulator of endothelial nitric oxide synthase and arterial stiffness in mice. Mol Endocrinol. 2014;28(1):53-64.

99. Li YC, Qiao G, Uskokovic M, Xiang W, Zheng W, Kong J. Vitamin D: a negative endocrine regulator of the renin-angiotensin system and blood pressure. J Steroid Biochem Mol Biol. 2004;8(9-90):387-392.

100. Sonderman JS, Munro HM, Blot WJ, Signorello LB. Reproducibility of serum 25-hydroxyvitamin D and vitamin D-binding protein levels over time in a prospective cohort study of black and white adults. $\mathrm{Am}$ J Epidemiol. 2012;176(7):615-621.
101. Hofmann JN, Yu K, Horst RL, Hayes RB, Purdue MP. Long-term variation in serum 25-hydroxyvitamin D concentration among participants in the prostate, lung, colorectal, and ovarian cancer screening trial. Cancer Epidemiol Biomarkers Prev. 2010;19(4):927-931.

102. Bolland MJ, Grey A, Reid IR. Differences in overlapping metaanalyses of vitamin D supplements and falls. J Clin Endocrinol Metab. 2014;99(11):4265-4272.

103. Vimaleswaran KS, Cavadino A, Berry DJ, et al. International Consortium for Blood Pressure (ICBP); Cohorts for Heart and Aging Research in Genomic Epidemiology (CHARGE) consortium; Global Blood Pressure Genetics (Global BPGen) consortium. Association of vitamin D status with arterial blood pressure and hypertension risk: a mendelian randomisation study. Lancet Diabetes Endocrinol. 2014;2(9):719-729.

104. Pittas AG, Chung M, Trikalinos T, et al. Systematic review: vitamin D and cardiometabolic outcomes. Ann Intern Med. 2010; 152(5):307-314.

105. Manson JE, Bassuk SS, Lee IM, et al. The VITamin D and OmegA-3 TriaL (VITAL): rationale and design of a large randomized controlled trial of vitamin D and marine omega-3 fatty acid supplements for the primary prevention of cancer and cardiovascular disease. Contemp Clin Trials. 2012;33(1):159-171. 


\section{Supplementary material PRISMA 2009 checklist}

\begin{tabular}{|c|c|c|}
\hline Section/topic & $\begin{array}{l}\text { Item } \\
\text { number }\end{array}$ & Checklist item \\
\hline Title & 1 & Identify the report as a systematic review, meta-analysis, or both \\
\hline \multicolumn{3}{|l|}{ Abstract } \\
\hline Structured summary & 2 & $\begin{array}{l}\text { Provide a structured summary including, as applicable, background, } \\
\text { objectives, data sources, study eligibility criteria, participants, } \\
\text { interventions, study appraisal and synthesis methods, results, } \\
\text { limitations, conclusions and implications of key findings, systematic } \\
\text { review registration number }\end{array}$ \\
\hline \multicolumn{3}{|r|}{ 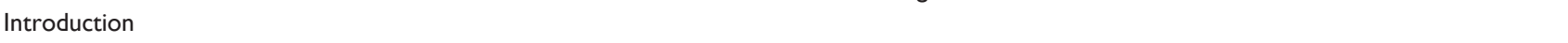 } \\
\hline Rationale & 3 & $\begin{array}{l}\text { Describe the rationale for the review in the context of what } \\
\text { is already known }\end{array}$ \\
\hline Objectives & 4 & $\begin{array}{l}\text { Provide an explicit statement of questions being addressed with } \\
\text { reference to Participants, Interventions, Comparisons and Outcomes, } \\
\text { Studies (PICOS) design }\end{array}$ \\
\hline \multicolumn{3}{|l|}{ Methods } \\
\hline Protocol and registration & 5 & $\begin{array}{l}\text { Indicate if a review protocol exists, if and where it can be accessed } \\
\text { (such as web address), and if available, provide registration information } \\
\text { including registration number }\end{array}$ \\
\hline Eligibility criteria & 6 & $\begin{array}{l}\text { Specify study characteristics (such as PICOS, length of follow-up) } \\
\text { and report characteristics (such as years considered, language, } \\
\text { publication status) used as criteria for eligibility, giving rationale }\end{array}$ \\
\hline Information sources & 7 & $\begin{array}{l}\text { Describe all information sources (such as databases with dates } \\
\text { of coverage, contact with study authors to identify additional studies) } \\
\text { in the search and date last searched }\end{array}$ \\
\hline Search & 8 & $\begin{array}{l}\text { Present full electronic search strategy for at least one database, } \\
\text { including any limits used, such that it could be repeated }\end{array}$ \\
\hline Study selection & 9 & $\begin{array}{l}\text { State the process for selecting studies (that is, screening, eligibility, } \\
\text { included in systematic review, and if applicable, included } \\
\text { in the meta-analysis) }\end{array}$ \\
\hline Data collection process & 10 & $\begin{array}{l}\text { Describe method of data extraction from reports (such as piloted } \\
\text { forms, independently, in duplicate) and any processes for obtaining } \\
\text { and confirming data from investigators }\end{array}$ \\
\hline Data items & 11 & $\begin{array}{l}\text { List and define all variables for which data were sought (such as } \\
\text { PICOS, funding sources) and any assumptions and simplifications made }\end{array}$ \\
\hline Risk of bias in individual studies & 12 & $\begin{array}{l}\text { Describe methods used for assessing risk of bias of individual studies } \\
\text { (including specification of whether this was done at the study or } \\
\text { outcome level), and how this information is to be used in any data } \\
\text { synthesis }\end{array}$ \\
\hline Summary measures & 13 & $\begin{array}{l}\text { State the principal summary measures (such as risk ratio, difference } \\
\text { in means) }\end{array}$ \\
\hline Synthesis of results & 14 & $\begin{array}{l}\text { Describe the methods of handling data and combining results of } \\
\text { studies, if done, including measures of consistency (such as } l^{2} \text { statistic) } \\
\text { for each meta-analysis }\end{array}$ \\
\hline Risk of bias across studies & 15 & $\begin{array}{l}\text { Specify any assessment of risk of bias that may affect the cumulative } \\
\text { evidence (such as publication bias, selective reporting within studies) }\end{array}$ \\
\hline Additional analyses & 16 & $\begin{array}{l}\text { Describe methods of additional analyses (such as sensitivity or subgroup } \\
\text { analyses, meta-regression), if done, indicating which were prespecified }\end{array}$ \\
\hline \multicolumn{3}{|r|}{ r } \\
\hline Study selection & 17 & $\begin{array}{l}\text { Give numbers of studies screened, assessed for eligibility, and included } \\
\text { in the review, with reasons for exclusions at each stage, ideally with } \\
\text { a flow diagram }\end{array}$ \\
\hline Study characteristics & 18 & $\begin{array}{l}\text { For each study, present characteristics for which data were extracted } \\
\text { (such as study size, PICOS, follow-up period) and provide the citations }\end{array}$ \\
\hline Risk of bias within studies & 19 & $\begin{array}{l}\text { Present data on risk of bias of each study and if available, } \\
\text { any outcome-level assessment (see item 12) }\end{array}$ \\
\hline
\end{tabular}


(Continued)

\begin{tabular}{|c|c|c|}
\hline Section/topic & $\begin{array}{l}\text { Item } \\
\text { number }\end{array}$ & Checklist item \\
\hline Results of individual studies & 20 & $\begin{array}{l}\text { For all outcomes considered (benefits or harms), present for each } \\
\text { study (a) simple summary data for each intervention group and (b) } \\
\text { effect estimates and confidence intervals, ideally with a forest plot }\end{array}$ \\
\hline Synthesis of results & 21 & $\begin{array}{l}\text { Present results of each meta-analysis done, including confidence } \\
\text { intervals and measures of consistency }\end{array}$ \\
\hline Risk of bias across studies & 22 & $\begin{array}{l}\text { Present results of any assessment of risk of bias across studies } \\
\text { (see item 15) }\end{array}$ \\
\hline Additional analysis & 23 & $\begin{array}{l}\text { Give results of additional analyses, if done (such as sensitivity or } \\
\text { subgroup analyses, meta-regression) (see item 16) }\end{array}$ \\
\hline \multicolumn{3}{|l|}{ Discussion } \\
\hline Summary of evidence & 24 & $\begin{array}{l}\text { Summarize the main findings including the strength of evidence for } \\
\text { each main outcome; consider their relevance to key groups (such as } \\
\text { health care providers, users, and policy makers) }\end{array}$ \\
\hline Limitations & 25 & $\begin{array}{l}\text { Discuss limitations at study and outcome level (such as risk of bias), } \\
\text { and at review level (such as incomplete retrieval of identified research, } \\
\text { reporting bias) }\end{array}$ \\
\hline Conclusions & 26 & $\begin{array}{l}\text { Provide a general interpretation of the results in the context } \\
\text { of other evidence, and implications for future research }\end{array}$ \\
\hline \multicolumn{3}{|l|}{ Funding } \\
\hline Funding & 27 & $\begin{array}{l}\text { Describe sources of funding for the systematic review and other } \\
\text { support (such as supply of data) and role of funders for the } \\
\text { systematic review }\end{array}$ \\
\hline
\end{tabular}

Abbreviation: PICOS, participants, interventions, comparisons, outcomes, and study.

\section{Publish your work in this journal}

Integrated Blood Pressure Control is an international, peer-reviewed open-access journal focusing on the integrated approach to managing hypertension and risk reduction. Treating the patient and comorbidities together with diet and lifestyle modification and optimizing healthcare resources through a multidisciplinary team approach constitute key

\section{Dovepress}

features of the journal. This journal is indexed on American Chemical Society's Chemical Abstracts Service (CAS). The manuscript management system is completely online and includes a very quick and fair peerreview system, which is all easy to use. Visit http://www.dovepress.com/ testimonials.php to read real quotes from published authors.

\footnotetext{
Submit your manuscript here: http://www.dovepress.com/integrated-blood-pressure-control-journal
} 